\title{
LOW-FREQUENCY RADIO CONTINUUM EVIDENCE FOR COOL IONIZED GAS IN NORMAL SPIRAL GALAXIES
}

\author{
F. P. ISRAEL \\ Sterrewacht Leiden, The Netherlands \\ AND \\ M. J. Mahoney \\ Clark Lake Radio Observatory, University of Maryland \\ Received 1988 January 18; accepted 1989 September 18
}

\begin{abstract}
We used the Clark Lake Radio Observatory TPT array at a frequency of $57.5 \mathrm{MHz}$ to survey a total of 133 galaxies, mostly late-type spirals; 68 galaxies were detected. Observed radio intensities $S_{\text {obs }}$ are systematically lower than intensities $S_{\text {extr }}$ extrapolated from measurements at higher frequencies. The ratio $S_{\text {obs }} / S_{\text {extr }}$ correlates well with the axial ratio of the observed galaxies and is smallest for edge-on galaxies. This is interpreted as increasing free-free absorption of nonthermal emission in galaxy disks with increasing tilt. The small ratio for edge-on galaxies also indicates that disk-dominated spiral galaxies generally do not have significant nonthermal halos.

The implied free-free absorption cannot not be caused by classical discrete $\mathrm{H}$ II regions. If it is to be explained by a smoothly distributed diffuse ionized gas component, this component should have a filling factor greater than 0.5 , and a very low electron temperature $T_{e} \approx 50 \mathrm{~K}$. We consider the more likely explanation to be the pervasive presence of a clumpy medium of well-mixed non-thermally emitting and thermally absorbing gas, with a small filling factor. With an electron temperature $500 \mathrm{~K} \leq T_{e} \leq 1000 \mathrm{~K}$ and a clump density of order $1 \mathrm{~cm}^{-3}$, ionization could be maintained by the normal OB star content of galaxies. There is at present no clear observational evidence for the presence of such a gas in our own Galaxy. If it exists, it should be located in the "thick disk." In particular, it should be mostly absent in the Galactic plane, and almost completely absent in the solar neighborhood.
\end{abstract}

Subject headings: galaxies: general — interstellar: matter - radio sources: galaxies — radio sources: spectra

\section{INTRODUCTION}

Since the radio detection of M31 by Hanbury Brown and Hazard (1951), a large number of galaxies has been observed at radio wavelengths. A listing of observations and results from the first two decades was published by Haynes et al. (1975). Most surveys were made using single-dish telescopes with beam sizes comparable to or larger than the observed galaxy sizes, yielding peak or integrated flux densities only. The advent of radio telescope arrays, notably aperture synthesis telescopes, opened the possibility of observing galaxies with substantially smaller beam sizes so that structural details could be studied. A useful, though now dated, review on the radio continuum morphology of spiral galaxies was given by van der Kruit and Allen (1976).

Observations of the integrated flux density of galaxies as a function of frequency yield the radio continuum spectrum, the shape of which is usually characterized by the spectral index $\alpha$ (defined as $S_{v} \propto v^{\alpha}$ ). This spectral index provides a clue to the nature of the radiation mechanism. For instance, optically thin thermal plasmas have $\alpha=-0.1$, while synchrotron emission is characterized by an appreciably steeper spectrum with $\alpha=-0.5$ to -1.0 , depending on the injection spectrum of energetic electrons and subsequent energy losses. Unfortunately, most galaxies have been observed reliably only in the range from 1 to $5 \mathrm{GHz}$; a limited number of galaxies have also been observed at frequencies as low as $408 \mathrm{MHz}$. Recent surveys (e.g., Gioia, Gregorini, and Klein 1982; Israel and Van der Hulst 1983) have extended this range to $10 \mathrm{GHz}$ on the high-frequency side. A mean spectral index $\alpha=-0.73$ has been found, although a number of galaxies show a spectral flattening at high frequencies (Israel and Van der Hulst 1983); this is ascribed to the increasing importance of optically thin radiation from ionized hydrogen (H II) regions with increasing observing frequency.

Very little is known about the radio spectrum of spiral galaxies at frequencies substantially lower than $408 \mathrm{MHz}$. For example, only a few galaxies are bright enough to be included in the $3 \mathrm{C}$ and $4 \mathrm{C}$ surveys at $178 \mathrm{MHz}$. At such low frequencies, single-dish telescopes generally have beam sizes too large to be of use. The large beam size raises the background confusion limit to flux values well above those of most normal galaxies. The only systematic very low frequency survey of a number of bright galaxies was made by Slee $(1972 a, b, 1977)$, who used the Culgoora radioheliograph at frequencies of 80 and $160 \mathrm{MHz}$ (beam sizes of 3.7 and 1'.8, respectively). A significant finding of that survey was the spectral flattening found in many galaxies at very low frequencies. Slee $(1972 b)$ suggested two possible explanations: a cutoff at low energies in the relativistic electron distribution, or free-free absorption by ionized gas in the galactic plane. However, from the published data, it cannot be excluded that in a number of galaxies a significant fraction of the extended disk emission was missed by the pencil beam used (e.g., NGC 253, NGC 1097, NGC 5236).

In this paper, we report the results of a $57.5 \mathrm{MHz}$ survey of 42 fields containing 133 galaxies, of which 68 were detected. This work was undertaken as part of a series of surveys at different radio frequencies aimed at establishing high-quality radio continuum spectra of spiral galaxies. The first paper in 
the series, dealing with observations at $0.43,0.83,5.0$, and 10.7 $\mathrm{GHz}$, was the survey by Israel and Van der Hulst (1983, hereafter Paper I)

\section{OBSERVATIONS}

We have used the broad-band Clark Lake Radio Observatory TPT synthesis radio telescope at a frequency of $57.5 \mathrm{MHz}$. This telescope consists of linear groupings (or banks) of $15 \mathrm{log}$ spiral elements each. There are 32 banks in the $300 \mathrm{~m}$ east-west arm of the T-shaped array and 16 banks in the $1800 \mathrm{~m}$ south arm. The fixed telescope is electronically pointed at a source by putting a linear phase gradient across the banks. This unique instrument is ideally suited ${ }^{\mathbf{1}}$ to our purpose because, at the observing frequency of $57.5 \mathrm{MHz}$, it allows instantaneous observation of a field of view of about $2.7 \times 2.7$ with a synthesized beam size of about 7' (somewhat dependent on source declination). This beam size is well-matched to the overall sizes of the sample galaxies, ensuring maximum sensitivity to galaxy emission. Maps for each field, corrected for instrumental effects, were made every 2-3 minutes and were visually examined for interference. Acceptable maps were added to produce a dirty map, which was then cleaned and restored. In about half the observed fields, comparison with the preliminary Texas $365 \mathrm{MHz}$ survey (J. N. Douglas, private communication) provided an additional check on source positions in the map and hence on systematic refraction during the observations. The telescope, its mode of operation, and its reduction procedures are described in more detail elsewhere (Erickson and Fisher 1974; Erickson, Mahoney, and Erb 1982).

The conversion of observed instrumental intensities to flux densities was done by frequent observation of calibrator sources (Table 1). The $57.5 \mathrm{MHz}$ flux densities of these sources are based on the spectra given by Kuhr (1980); the flux density scale used is that of Baars et al. (1977). In the reduction stage, a strong dependence of apparent flux density on zenith distance was noted and corrected for. All but five fields were reobserved on different nights; repeatability in virtually all cases was within $10 \%$

A list of the observed galaxy fields is given in Table 2 . Fields were generally centered on a bright galaxy and observed about transit. Column (4) of Table 2 gives the run number. Run 1

\footnotetext{
${ }^{1}$ Unfortunately, no longer. The Clark Lake Radio Observatory was closed down in 1987 due to lack of funding.
}

TABLE 1

\begin{tabular}{|c|c|}
\hline \multicolumn{2}{|c|}{ CALIBRATORS } \\
\hline Name & $\begin{array}{c}\text { Flux Density } \\
S_{57.5}(\mathrm{Jy})\end{array}$ \\
\hline $3 C 33$. & $128 \pm 18$ \\
\hline $3 \mathrm{C} 47$. & $92 \pm 13$ \\
\hline 3C 79 . & $79 \pm 8$ \\
\hline 3 C 98 & $118 \pm 7$ \\
\hline $3 \mathrm{C} 109$ & $60 \pm 6$ \\
\hline $3 \mathrm{C} 123$ & $460 \pm 30$ \\
\hline 3C 144 (Tau A) & $2450 \pm 280$ \\
\hline $3 \mathrm{C} 218(\operatorname{Hyd} A)$. & $774^{-}$ \\
\hline 3С $295 \ldots \ldots \ldots$ & $35 \pm 2$ \\
\hline $3 \mathrm{C} 274$ (Vir A) & $3285 \pm 415$ \\
\hline $3 C 296 \ldots \ldots$ & $35 \pm 7$ \\
\hline $3 C 313$ & $63 \pm 23$ \\
\hline $3 C 315$ & $62 \pm 15$ \\
\hline $3 \mathrm{C} 348(\operatorname{Her} \mathrm{A})$ & $1108 \pm 120$ \\
\hline
\end{tabular}

TABLE 2

FieLdS OBSERVEd at $57.5 \mathrm{MHz}$

\begin{tabular}{|c|c|c|c|c|c|}
\hline $\begin{array}{c}\text { Field } \\
\text { Number } \\
\text { (1) }\end{array}$ & $\begin{array}{l}\text { R.A. (1950) } \\
\text { (2) }\end{array}$ & $\begin{array}{l}\text { Decl. (1950) } \\
\text { (3) }\end{array}$ & $\begin{array}{c}\text { Run } \\
\text { (4) }\end{array}$ & $\begin{array}{c}\text { Beam Size } \\
\text { (arcminutes) } \\
\text { (5) }\end{array}$ & $\begin{array}{l}T_{\text {int }} \\
(\mathrm{hr}) \\
(6)\end{array}$ \\
\hline & $00^{\mathrm{h}} 45^{\mathrm{m}} 07^{\mathrm{s}}$ & $-25^{\circ} 34^{\prime} 0$ & 3 & $7.0 \times 11.0$ & 1 \\
\hline & 013400 & +1531.6 & 1,3 & $7.0 \times 6.0$ & 2.5 \\
\hline $3 .$. & $\begin{array}{lll}02 & 19 & 25\end{array}$ & +4207.2 & 1,3 & $7.0 \times 6.0$ & 2 \\
\hline & $\begin{array}{lll}02 & 40 & 07\end{array}$ & -0013.5 & 3 & $7.0 \times 7.0$ & 1 \\
\hline & $\begin{array}{lll}03 & 41 \quad 57\end{array}$ & +6757.0 & 3 & $7.0 \times 7.0$ & 1 \\
\hline & 042605 & +6444.4 & 1 & $6.5 \times 6.5$ & 4.5 \\
\hline & 050550 & -3735.9 & 1 & $7.0 \times 16.9$ & 1 \\
\hline $8 \ldots$ & 053634 & +6921.3 & 1 & $6.5 \times 6.5$ & 4.5 \\
\hline $9 \ldots \ldots \ldots$ & 061045 & +7822.5 & 1 & $7.0 \times 7.5$ & 4.5 \\
\hline $10 \ldots$ & 073200 & $\begin{array}{r}+6542.7 \\
\end{array}$ & 1 & $7.0 \times 6.5$ & 4.5 \\
\hline $11 .$. & $\begin{array}{lll}09 & 16 & 18\end{array}$ & +64 19.1 & 1 & $7.0 \times 6.0$ & 3 \\
\hline 12. & $\begin{array}{lll}09 & 18 & 36\end{array}$ & +5111.3 & 1 & $7.0 \times 6.0$ & 4.5 \\
\hline $13 \ldots$ & $\begin{array}{lll}09 & 29 & 20\end{array}$ & +2143.2 & 1 & $7.0 \times 6.0$ & 4.5 \\
\hline $14 \ldots$ & $\begin{array}{lll}09 & 39 & 58\end{array}$ & +3204.0 & 1 & $7.0 \times 6.0$ & 3 \\
\hline $15 .$. & 095136 & +6936.6 & 1 & $7.0 \times 6.5$ & 2 \\
\hline $16 \ldots$ & $\begin{array}{lll}09 & 48 & 36\end{array}$ & +5555.4 & 1 & $7.0 \times 6.0$ & 3 \\
\hline $17 \ldots$ & 102000 & +2100.0 & 1 & $7.0 \times 6.0$ & 4.5 \\
\hline $18 .$. & 103542 & +5346.0 & 2 & $7.0 \times 6.0$ & 3 \\
\hline $19 .$. & $1041 \quad 18$ & +1158.0 & 2 & $7.0 \times 6.5$ & 3 \\
\hline 20. & $\begin{array}{lll}11 & 03 & 12\end{array}$ & +0014.0 & 2 & $7.0 \times 7.0$ & 3 \\
\hline $21 \ldots$ & 110842 & +5557.0 & 2 & $7.0 \times 6.0$ & 3 \\
\hline $22 .$. & $\begin{array}{lll}11 & 17 & 39\end{array}$ & +1335.0 & 2 & $7.0 \times 6.0$ & 3 \\
\hline 23. & $\begin{array}{lll}11 & 59 & 18\end{array}$ & +1835.6 & 2 & $7.0 \times 9.5$ & 3 \\
\hline $24 \ldots$ & 115700 & +5215.0 & 1 & $7.0 \times 5.5$ & 2 \\
\hline $25 \ldots$ & 120300 & $\begin{array}{r}+5049.0 \\
\end{array}$ & 2 & $7.0 \times 6.0$ & 3 \\
\hline $26 .$. & $\begin{array}{lll}12 & 14 & 18\end{array}$ & +6945.0 & 2 & $7.0 \times 6.5$ & 3 \\
\hline $27 \ldots$ & $1216 \quad 28$ & +4735.0 & 2 & $7.0 \times 6.0$ & 3 \\
\hline 28 . & 121800 & +1525.0 & 2 & $7.0 \times 6.0$ & 3 \\
\hline $29 \ldots \ldots \ldots$ & $\begin{array}{lll}12 & 28 & 09\end{array}$ & +4155.0 & 2 & $7.0 \times 5.5$ & 3 \\
\hline $30 .$. & 124040 & +3237.0 & 1 & $7.0 \times 5.5$ & 1 \\
\hline $31 .$. & $1248 \quad 37$ & +4123.0 & 2 & $7.0 \times 5.5$ & 3 \\
\hline $32 \ldots$ & $\begin{array}{lll}13 & 03 & 30\end{array}$ & +3700.0 & 1 & $7.0 \times 5.5$ & 4 \\
\hline $33 \ldots$ & $\begin{array}{lll}13 & 11 & 13\end{array}$ & +3651.0 & 2 & $7.0 \times 5.5$ & 3 \\
\hline $34 \ldots$ & 131330 & +4217.0 & 2 & $7.0 \times 5.5$ & 3 \\
\hline $35 \ldots$ & 132748 & +4727.0 & 2 & $7.0 \times 6.0$ & 3 \\
\hline $36 \ldots$ & $\begin{array}{lll}13 & 34 & 18\end{array}$ & +2937.0 & 2 & $7.0 \times 13.0$ & 3 \\
\hline $37 \ldots \ldots \ldots$ & 140124 & +5435.6 & 2 & $7.0 \times 6.0$ & 3 \\
\hline $38 \ldots$. & 145130 & +0345.0 & 2 & $7.0 \times 6.5$ & 3 \\
\hline $39 \ldots$ & 151436 & +5631.0 & 2 & $7.0 \times 6.0$ & 4.5 \\
\hline $40 \ldots$ & 194206 & +1453.0 & 1 & $7.0 \times 8.0$ & 4.5 \\
\hline $41 .$. & $\begin{array}{lll}20 & 33 & 54\end{array}$ & +5958.0 & 2 & $6.5 \times 6.0$ & 3 \\
\hline $42 \ldots \ldots \ldots$ & $2234 \quad 10$ & +3350.0 & 2 & $6.5 \times 6.5$ & 3 \\
\hline
\end{tabular}

took place in 1985 February, Run 2 took place in 1985 May, and Run 3 took place in 1986 January. Column (5) gives the restoring beam size of each field; column (6) lists the total integration time per field in hours. The results on galaxies included in the observed fields are given in Tables 3 and 4 . In both tables, the first four columns give galaxy identification, optical position, and galaxy type taken from de Vaucouleurs, de Vaucouleurs, and Corwin (1976); column (5) identifies the field in which the galaxy was seen, and column (6) gives the integrated $57.5 \mathrm{MHz}$ flux density. Confusion limits were estimated from the maps and vary from 0.2 to $0.5 \mathrm{Jy}$. Sample fields are shown Figure 1.

\section{ANALYSIS}

\section{a) Comparison of Observed and Extrapolated Flux Densities}

We have used the extensive radio continuum data base available in the literature to extrapolate the observed radio continuum spectrum between centimeter and meter wavelengths to $57.5 \mathrm{MHz}$. Data relevant to this extrapolation are given in Table 5. Column (2) gives the best values for the 1.4 
ISRAEL AND MAHONEY

Vol. 352

TABLE 3

Galaxies Detected at $57.5 \mathrm{MHz}$

\begin{tabular}{|c|c|c|c|c|c|c|}
\hline $\begin{array}{c}\text { Name } \\
(1)\end{array}$ & $\begin{array}{l}\text { R.A. (1950) } \\
\text { (2) }\end{array}$ & $\begin{array}{c}\text { Decl. (1950) } \\
\text { (3) }\end{array}$ & $\begin{array}{c}\text { Galaxy } \\
\text { Type } \\
\text { (4) }\end{array}$ & $\begin{array}{c}\text { Field } \\
\text { Number } \\
(5)\end{array}$ & $\begin{array}{c}S_{57.5} \\
(\mathrm{Jy}) \\
(6)\end{array}$ & $\begin{array}{c}\text { Remarks } \\
(7)\end{array}$ \\
\hline NGC 253 & $00^{\mathrm{h}} 45^{\mathrm{m}} 08^{\mathrm{s}}$ & $-25^{\circ} 33^{\prime} 42^{\prime \prime}$ & Scp & 1 & $48 \pm 9$ & \\
\hline NGC $628 \ldots$ & 013401 & +153136 & Sc I & 2 & $2 \pm 1$ & M74 \\
\hline NGC $891 \ldots$. & 021925 & +420712 & $\mathrm{Sb} I$ & 3 & $6.6 \pm 1.8$ & \\
\hline NGC $1055 \ldots$ & 023911 & +001342 & Sb II-III & 4 & $1.3 \pm 0.5$ & \\
\hline NGC $1068 \ldots$ & $0240 \quad 07$ & -001330 & Sbp & 4 & $39 \pm 8$ & M77 \\
\hline IC $342 \ldots \ldots$. & 034158 & +675624 & Sc I-II & 5 & $8 \pm 3$ & \\
\hline NGC $1569 \ldots$ & 042605 & +644424 & Irp III-IV & 6 & $1.6 \pm 0.5$ & VII Zw16 \\
\hline NGC $1808 \ldots$. & 050559 & -373442 & SABa & 7 & $16 \pm 8$ & \\
\hline NGC $1961 \ldots$ & 053634 & +692118 & Sbpnt I & 8 & $2.0 \pm 0.5$ & \\
\hline NGC $2146 \ldots$ & 061045 & +782230 & Snt & 9 & $6.9 \pm 1.7$ & \\
\hline NGC $2403 \ldots$ & 073203 & +654242 & Sc II-III & 10 & $3 \pm 1.5$ & \\
\hline NGC $2805 \ldots$ & $0916 \quad 17$ & +641906 & SABd & 11 & $0.7 \pm 0.3$ & \\
\hline NGC $2814 \ldots$ & 091708 & +642754 & Ir & 11 & $0.2 \pm 0.2$ & \\
\hline NGC $2820 \ldots$ & 091735 & +6428 & $\mathrm{SBc}$ & 11 & $0.4 \pm 0.2$ & + IC 2458 \\
\hline NGC $2841 \ldots$ & $09 \quad 18 \quad 35$ & +511118 & $\mathrm{Sb} \mathrm{I}$ & 12 & $0.7 \pm 0.3$ & \\
\hline NGC $2903 \ldots$ & 092920 & +214312 & Sb I-II & 13 & $2.6 \pm 1.2$ & \\
\hline NGC $2916 \ldots$ & 093208 & +215548 & $\mathrm{Sb}$ & 13 & $0.2 \pm 0.2$ & Mrk 404 \\
\hline NGC $2964 \ldots$ & 093958 & +320424 & Scn II & 14 & $1.1 \pm 0.3$ & \\
\hline NGC 2959/61 & 094106 & +6850 & $\mathrm{Spb} / \mathrm{Sb}$ & 15 & $0.6 \pm 0.2$ & \\
\hline NGC $3031 \ldots$ & 095130 & +691828 & Sb I-II & 15 & $2.4 \pm 0.6$ & M81 \\
\hline NGC $3034 \ldots$ & 095141 & +695454 & Irp & 15 & $29 \pm 6$ & M82 \\
\hline NGC $3079 \ldots$ & $0958 \quad 35$ & +555524 & Sbnt II & 16 & $6.9 \pm 1.2$ & \\
\hline NGC $3190 \ldots$. & 101520 & +220506 & Sbnt II-III & 17 & $0.3 \pm 0.2$ & \\
\hline NGC $3213 \ldots$ & $1018 \quad 34$ & +195418 & Sc & 17 & $0.3 \pm 0.2$ & \\
\hline NGC $3221 \ldots$. & 101935 & +214906 & SBc & 17 & $0.3 \pm 0.2$ & \\
\hline NGC $3227 / 6$. & 102045 & +2008 & Sbnt/E2 & 17 & $0.3 \pm 0.2$ & \\
\hline IC $610 \ldots \ldots$. & 102345 & +202900 & Sbc & 17 & $0.4 \pm 0.2$ & \\
\hline NGC $3310 \ldots$ & 103539 & +534554 & Ir II & 18 & $2.8 \pm 0.8$ & \\
\hline NGC $3351 \ldots$. & $1041 \quad 19$ & +115806 & S(B)b II & 19 & $1.2 \pm 0.4$ & M95 \\
\hline NGC $3521 \ldots$. & 110316 & +001412 & Sb II & 20 & $13 \pm 2.0$ & \\
\hline NGC $3556 \ldots$ & 110849 & +555642 & Sc & 21 & $1.8 \pm 0.5$ & M108 \\
\hline NGC $3627 \ldots$ & 111738 & +131518 & Sbn II & 22 & $2.3 \pm 0.7$ & M66 \\
\hline NGC $3628 \ldots$ & 111740 & +135206 & Sbnt & 22 & $2.9 \pm 0.7$ & \\
\hline NGC $3953 \ldots$. & $1151 \quad 13$ & +523630 & Sb I & 23 & $0.9 \pm 0.5$ & \\
\hline NGC $4027 \ldots$ & 115656 & -185924 & Sct & 24 & $1.3 \pm 0.5$ & \\
\hline NGC 4038/9 . & 115920 & -183542 & $\mathrm{P}$ & 24 & $3.5 \pm 0.8$ & \\
\hline NGC $4088 \ldots$ & 120303 & +504912 & Sc I-II & 25 & $1.4 \pm 0.4$ & \\
\hline NGC $4100 \ldots$ & 120336 & +495136 & Sb I-II & 25 & $1.0 \pm 0.4$ & \\
\hline NGC $4102 \ldots$ & 120352 & +522918 & Sct & 23 & $2.0 \pm 0.6$ & \\
\hline NGC $4120 \ldots$ & 120604 & +694924 & $\mathrm{Sc}$ & 26 & $0.4 \pm 0.2$ & \\
\hline NGC $4157 \ldots$ & 120834 & +504542 & $\mathrm{Sb}$ II & 25 & $0.7 \pm 0.2$ & \\
\hline NGC $4217 \ldots$ & $\begin{array}{lll}12 & 13 & 21\end{array}$ & +472218 & $\mathrm{Sb}$ & 27 & $0.3 \pm 0.2$ & \\
\hline NGC $4254 \ldots$ & $\begin{array}{lll}12 & 16 & 17\end{array}$ & +144142 & Sc I & 28 & $5.8 \pm 1.0$ & M99 \\
\hline NGC $4258 \ldots$. & 121629 & +473500 & Sbp & 27 & $5.6 \pm 1.0$ & M106 \\
\hline IC 783A $\ldots . .$. & 121948 & +160036 & L & 28 & $0.3 \pm 0.3$ & \\
\hline NGC $4321 \ldots$ & 122023 & +160600 & Sc I & 28 & $2.3 \pm 0.6$ & M100 \\
\hline NGC $4346 \ldots$ & 122102 & +471612 & E6 & 27 & $1.3 \pm 0.3$ & \\
\hline NGC $4490 / 85$ & 122810 & +415454 & Scn III & 29 & $5.0 \pm 0.6$ & \\
\hline NGC $4631 / 27 \ldots \ldots \ldots \ldots$ & 123941 & +324848 & Sc III & 30 & $6.3 \pm 2.1$ & \\
\hline NGC 4656/7 ................ & 124133 & +322700 & Sct IV & 30 & $0.5 \pm 0.3$ & \\
\hline NGC $4736 \ldots \ldots \ldots \ldots \ldots$ & 124832 & +412336 & Sbp II & 31 & $1.4 \pm 0.5$ & M94 \\
\hline NGC $5005 \ldots \ldots \ldots \ldots \ldots$ & 130837 & +371924 & $\mathrm{Sb}$ III & $32 / 33$ & $1.6 \pm 0.3$ & \\
\hline NGC $5014 \ldots \ldots \ldots \ldots \ldots$ & 130913 & +363300 & $\mathbf{S}$ & 33 & $1.1 \pm 0.4$ & \\
\hline NGC $5033 \ldots \ldots \ldots \ldots \ldots$ & 131108 & +365148 & Sb I-II & 33 & $1.6 \pm 0.6$ & \\
\hline NGC $5055 \ldots$. & 131335 & +421748 & $\mathrm{Sb}$ II & 34 & $2.1 \pm 0.5$ & M63 \\
\hline NGC $5194 / 5 \ldots \ldots \ldots \ldots \ldots$ & 132746 & +472718 & Sc I & 35 & $11 \pm 1.5$ & \\
\hline NGC $5236 \ldots$ & 133410 & -293648 & Sc I-II & 36 & $29 \pm 5$ & M83 \\
\hline NGC $5457 \ldots$. & 140127 & +543536 & Sc I & 37 & $6.5 \pm 2.5$ & M101 \\
\hline NGC $5477 \ldots \ldots \ldots \ldots \ldots$ & 140348 & +544206 & Ir IV-V & 37 & $0.3 \pm 0.2$ & \\
\hline NGC $5484 / 5 \ldots \ldots \ldots \ldots \ldots$ & 140515 & +5515 & $\mathrm{E} 2 / \mathrm{S} 0$ & 37 & $1.4 \pm 0.4$ & \\
\hline NGC 5775/4 & 145118 & +0345 & $\mathrm{Sb} / \mathrm{S}$ & 38 & $2.5 \pm 1.0$ & \\
\hline NGC $5879 \ldots$. & 150829 & +571124 & $\mathrm{Sb}$ II-III & 39 & $0.3 \pm 0.2$ & \\
\hline NGC $5907 \ldots \ldots \ldots \ldots \ldots$ & 151437 & +563024 & $\mathrm{Sb}$ II & 39 & $2.3 \pm 0.5$ & \\
\hline NGC $5908 \ldots$. & 151523 & +553530 & $\mathrm{Sb}$ & 39 & $0.4 \pm 0.2$ & \\
\hline NGC $6822 \ldots \ldots \ldots \ldots \ldots$ & 194207 & -145542 & Ir IV-V & 40 & & \\
\hline NGC $6946 \ldots \ldots \ldots \ldots \ldots$ & 203348 & +595900 & Sc I & 41 & $13 \pm 2$ & \\
\hline NGC 7317-20 ............. & 223340 & +3342 & $\mathrm{E} / \mathrm{S}$ & 42 & $1.0 \pm 0.4$ & \\
\hline NGC $7331 \ldots \ldots \ldots \ldots \ldots$ & $2234 \quad 47$ & +340930 & Sb I-II & 42 & $4.5 \pm 1.3$ & \\
\hline
\end{tabular}


TABLE 4

GaLAXY UPPER LiMits AT $57.5 \mathrm{MHz}$

\begin{tabular}{|c|c|c|c|c|c|c|}
\hline $\begin{array}{l}\text { Name } \\
(1)\end{array}$ & $\begin{array}{l}\text { R.A. (1950) } \\
\text { (2) }\end{array}$ & $\begin{array}{l}\text { Decl. (1950) } \\
\text { (3) }\end{array}$ & $\begin{array}{c}\text { Galaxy } \\
\text { Type } \\
\text { (4) }\end{array}$ & $\begin{array}{c}\text { Field } \\
\text { Number } \\
(5)\end{array}$ & $\begin{array}{l}S_{57.5} \\
(\mathrm{Jy}) \\
(6)\end{array}$ & $\begin{array}{c}\text { Remarks } \\
(7)\end{array}$ \\
\hline NGC $898 \ldots$ & $02^{\mathrm{h}} 20^{\mathrm{m}} 11^{\mathrm{s}}$ & $+41^{\circ} 43^{\prime} 30^{\prime \prime}$ & $\mathrm{Sb}$ & 3 & $<0.7$ & \\
\hline NGC $906 \ldots \ldots$ & 022206 & +4152 & Sab & 3 & $<0.7$ & \\
\hline NGC $910 \ldots \ldots \ldots \ldots \ldots \ldots$ & $02 \quad 2218$ & +413606 & $\mathrm{~L}$ & 3 & $<0.7$ & \\
\hline NGC $1032 \ldots$ & 023648 & +0053 & $\mathrm{Sa}$ & 4 & $<1.0$ & Possible Detection \\
\hline NGC 1072 . & 024057 & +000542 & $\mathrm{Sb}$ & 4 & $<0.6$ & \\
\hline NGC $1087 \ldots$ & 024351 & -004230 & Sc III & 4 & $<0.7$ & Possible Detection \\
\hline NGC $1090 \ldots$. & 024406 & -002724 & Sn IV & 4 & $<0.6$ & \\
\hline NGC 1094 & 024455 & -002930 & Sab & 4 & $<0.6$ & \\
\hline NGC 2146A & $0615 \quad 53$ & +783318 & $\mathrm{Sc}$ & 9 & $<0.4$ & \\
\hline NGC $2944 \ldots .$. & 093621 & +323218 & $\mathrm{SBc}$ & 14 & $<0.3$ & \\
\hline NGC 2970 . & $0940 \quad 33$ & +321230 & E & 14 & $<0.3$ & Mrk 405 \\
\hline NGC 3073 . & 095729 & +555136 & $\mathrm{~L}$ & 15 & $<0.4$ & Mrk 131 \\
\hline NGC 3077 & $\begin{array}{lll}09 & 59 & 24\end{array}$ & +685830 & E2p & 15 & $<0.5$ & Possible Detection \\
\hline NGC $3185 \ldots \ldots \ldots \ldots \ldots$ & 101453 & +215618 & S(B)b III & 17 & $<0.3$ & Possible Detection \\
\hline NGC 3187 & 101502 & +220730 & SBc & 17 & $<0.2$ & \\
\hline NGC 3193 & 101539 & +220848 & E0 & 17 & $<0.2$ & \\
\hline NGC $3222 \ldots$ & 101950 & +200824 & $\mathrm{~L}$ & 17 & $<0.2$ & \\
\hline NGC $3368 \ldots \ldots \ldots \ldots \ldots$ & 104407 & +120506 & Spb & 19 & $<0.3$ & \\
\hline NGC $3379 \ldots \ldots$. & 104511 & +125048 & E1 & 19 & $<0.3$ & \\
\hline NGC $3384 \ldots .$. & 104538 & +125342 & E7 & 19 & $<0.3$ & \\
\hline NGC $3389 \ldots \ldots .$. & $1045 \quad 50$ & +124754 & Sc III & 19 & $<0.3$ & \\
\hline NGC $3499 \ldots \ldots \ldots \ldots \ldots$ & 110013 & +562930 & Ir & 21 & $<0.3$ & \\
\hline NGC $3517 \ldots \ldots$. & 110238 & +564742 & $\mathrm{Sb}$ & 21 & $<0.3$ & \\
\hline NGC $3623 \ldots$ & $11 \quad 1619$ & +132154 & Sbn II & 22 & $<0.3$ & \\
\hline NGC $4024 \ldots \ldots \ldots \ldots \ldots$ & 115558 & -180412 & E2 & 23 & $<0.8$ & \\
\hline NGC $4026 \ldots \ldots \ldots \ldots \ldots$ & 115650 & +511424 & E8 & 24,25 & $<0.4$ & \\
\hline NGC $4033 \ldots$ & 115891 & -173354 & E5 & 23 & $<0.8$ & \\
\hline NGC $4068 \ldots \ldots \ldots \ldots \ldots$ & 120128 & +525200 & Im & 24 & $<0.5$ & \\
\hline NGC $4085 \ldots \ldots \ldots \ldots \ldots$ & 120251 & +503756 & Sb III & 25 & $<0.3$ & \\
\hline NGC $4128 \ldots \ldots \ldots \ldots \ldots$ & 120604 & +690248 & So & 26 & $<0.3$ & \\
\hline NGC $4218 \ldots \ldots \ldots \ldots \ldots$ & $1213 \quad 17$ & +482430 & $\mathrm{Sa}$ & 27 & $<0.3$ & \\
\hline NGC $4220 \ldots \ldots \ldots$ & 121343 & +480930 & So & 27 & $<0.3$ & \\
\hline NGC $4226 \ldots \ldots \ldots \ldots \ldots$ & 121357 & +471812 & $\mathrm{Sa}$ & 27 & $<0.3$ & \\
\hline NGC 4231/2 $\ldots \ldots \ldots \ldots \ldots$ & 121420 & +4744 & $\mathrm{~L} / \mathrm{Sbc}$ & 27 & $<0.3$ & \\
\hline NGC $4236 \ldots \ldots \ldots \ldots \ldots$ & $1214 \quad 19$ & +694500 & SB IV & 26 & $<1.5$ & \\
\hline NGC $4248 \ldots \ldots \ldots \ldots \ldots$ & 121521 & +474112 & Ir & 27 & $<0.3$ & \\
\hline NGC $4262 \ldots \ldots \ldots \ldots \ldots$ & 121657 & +150918 & E1 & 28 & $<0.3$ & \\
\hline NGC $4288 \ldots \ldots \ldots \ldots \ldots$ & $1218 \quad 10$ & +463406 & S(B) IV-V & 27 & $<0.3$ & DDO 119 \\
\hline NGC $4302 \ldots \ldots \ldots \ldots \ldots$ & $12 \quad 19 \quad 10$ & +145236 & $\mathrm{Sc}$ & 28 & $<0.3$ & \\
\hline NGC $4312 \ldots \ldots$. & 121959 & +154900 & Sab & 28 & $<0.3$ & \\
\hline NGC $4328 \ldots \ldots \ldots \ldots \ldots$ & $12 \quad 2048$ & +1605 & $\mathrm{~L}$ & 28 & $<0.3$ & \\
\hline NGC 4377 . & $12 \quad 2241$ & +150224 & E1 & 28 & $<0.3$ & \\
\hline NGC $4704 \ldots \ldots \ldots \ldots \ldots$ & 124625 & +421142 & SBbcp & 31 & $<0.3$ & \\
\hline NGC $4914 \ldots \ldots \ldots \ldots \ldots$ & 125822 & +373506 & $\ldots$ & 32 & $<0.3$ & \\
\hline IC $4182 \ldots \ldots \ldots \ldots \ldots$ & $1303 \quad 31$ & +375230 & Sm & 32 & $<0.3$ & \\
\hline IC 4189 .................... & 130342 & +3614 & Scd & 32 & $<0.3$ & \\
\hline NGC $5169 \ldots \ldots \ldots \ldots \ldots$ & 132604 & +465554 & $\mathrm{SBb}$ & 35 & $<0.3$ & \\
\hline NGC $5173 \ldots \ldots \ldots \ldots \ldots$ & 132619 & +465106 & $\mathrm{E}$ & 35 & $<0.3$ & \\
\hline IC $4263 \ldots \ldots \ldots \ldots \ldots$ & 132626 & +471106 & SBd & 35 & $<0.3$ & \\
\hline NGC $5198 \ldots \ldots \ldots \ldots \ldots \ldots$ & 132804 & +465542 & E2 & 35 & $<0.3$ & I Zw 59 \\
\hline NGC $5229 \ldots \ldots \ldots \ldots \ldots$ & 133159 & +481018 & SBd & 35 & $<0.3$ & \\
\hline NGC $5264 \ldots \ldots \ldots \ldots \ldots \ldots$ & 133847 & -293942 & Ir IV-V & 36 & $<2.7$ & DDO 242 \\
\hline NGC $5422 \ldots \ldots \ldots \ldots \ldots \ldots$ & 135856 & +552418 & E9 & 37 & $<0.3$ & \\
\hline NGC $5473 \ldots \ldots \ldots \ldots \ldots$ & 140259 & +550754 & E2 & 37 & $<0.3$ & \\
\hline NGC $5474 \ldots \ldots \ldots \ldots \ldots$ & 140316 & +535406 & Scnt & 37 & $<0.3$ & \\
\hline NGC $5486 \ldots \ldots \ldots \ldots \ldots$ & 140541 & +552018 & $\mathrm{Sm}$ & 37 & $<0.3$ & \\
\hline IC $1067 \ldots \ldots \ldots \ldots \ldots$ & 145034 & +033212 & SBb & 38 & $<0.4$ & \\
\hline NGC 5770 & 145045 & +040950 & $\ldots$ & 38 & $<0.4$ & \\
\hline IC $1099 \ldots \ldots \ldots \ldots \ldots \ldots$ & 150535 & +564200 & SBbc & 39 & $<0.3$ & \\
\hline NGC 5866B $\ldots \ldots \ldots \ldots \ldots$ & 151046 & +555824 & $\mathrm{Sd}$ & 39 & $<0.3$ & \\
\hline NGC $5905 \ldots \ldots \ldots \ldots \ldots$ & 151403 & +554206 & $\mathrm{~S}(\mathrm{~B}) \mathrm{b} \mathrm{I}$ & 39 & $<0.3$ & \\
\hline NGC $7315 \ldots \ldots \ldots \ldots \ldots \ldots$ & $2233 \quad 15$ & +343236 & $\ldots$ & 42 & $<0.5$ & \\
\hline NGC $7335 \ldots \ldots \ldots \ldots \ldots$ & 223502 & +341118 & L & 42 & $<0.5$ & \\
\hline NGC $7337 \ldots \ldots \ldots \ldots \ldots$ & 223509 & +340648 & SBb II & 42 & $<0.5$ & \\
\hline NGC $7343 \ldots \ldots \ldots \ldots \ldots \ldots$ & $2236 \quad 19$ & +334830 & SBbc & 42 & $<0.5$ & \\
\hline
\end{tabular}



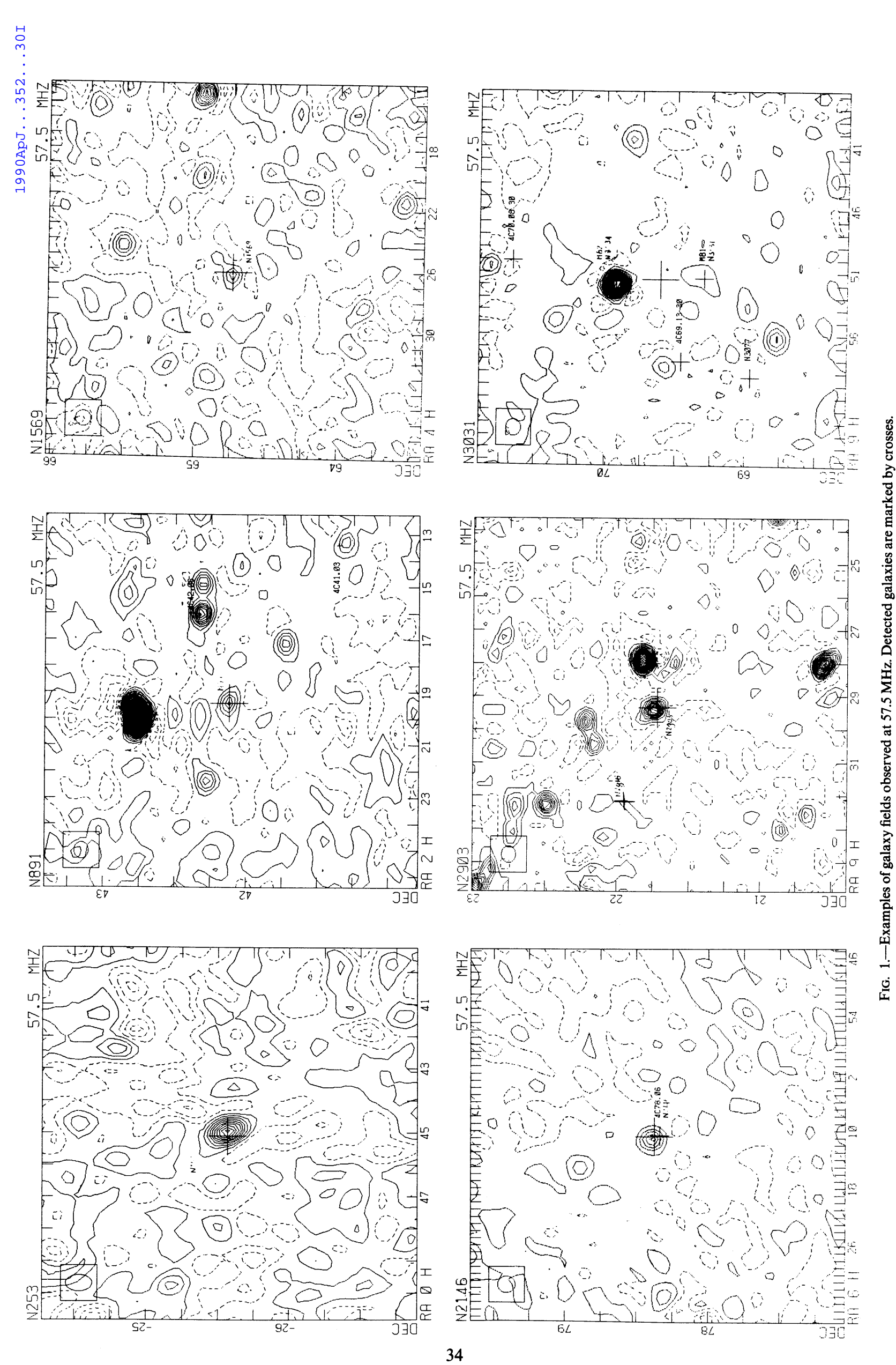

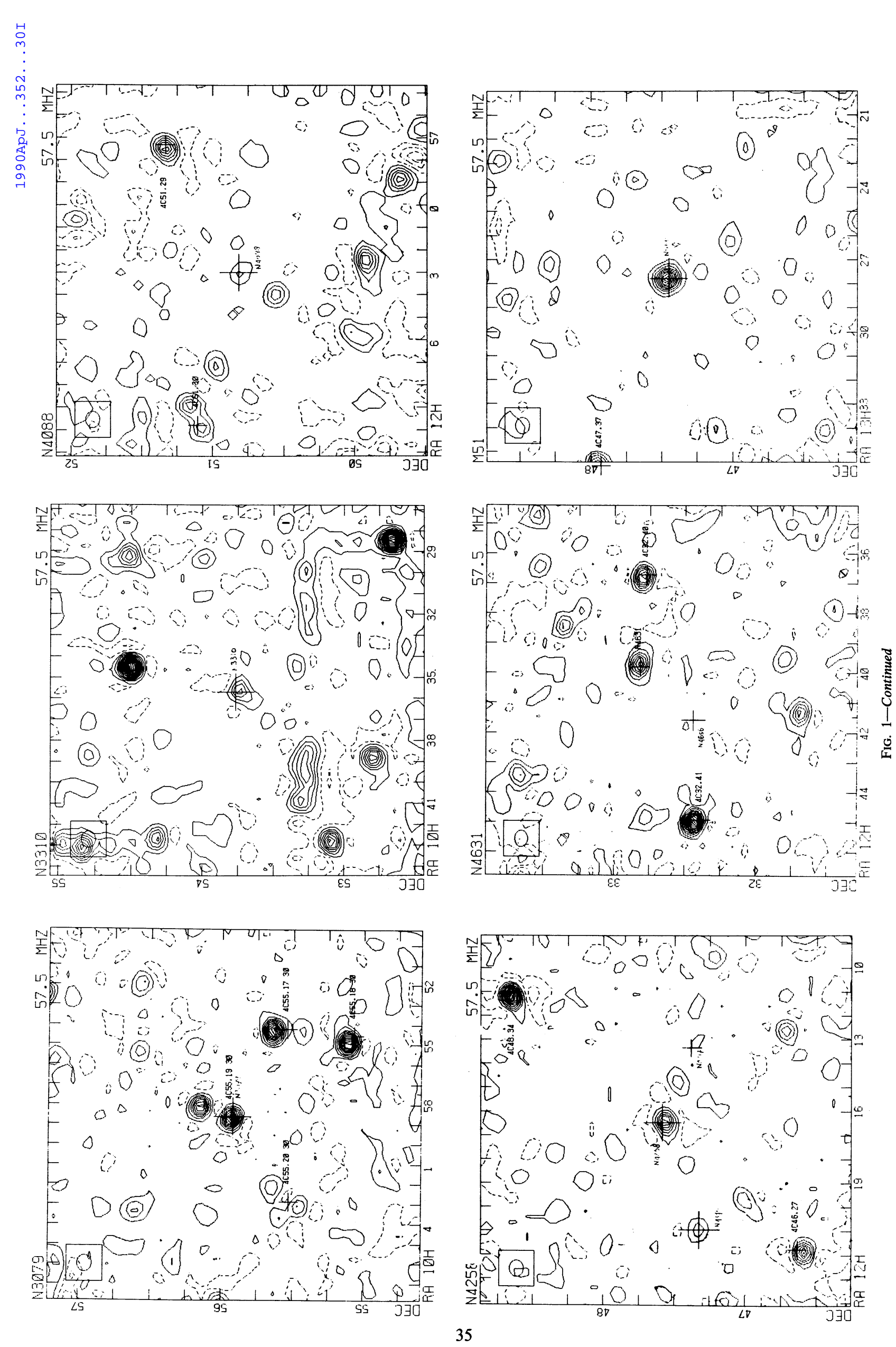
TABLE 5

Previously Observed and Extrapolated Flux Densities

\begin{tabular}{|c|c|c|c|c|c|c|}
\hline \multirow[b]{2}{*}{$\begin{array}{c}\text { NAME } \\
\text { (1) }\end{array}$} & \multicolumn{3}{|c|}{ VALUES USED IN ANALYSIS } & \multirow{2}{*}{$\begin{array}{c}\text { EXTRAPOLATED } \\
\text { FLUX DENSITY } \\
S_{57.5} \\
(\mathbf{J y}) \\
(5)\end{array}$} & \multirow{2}{*}{$\begin{array}{c}\text { OBSERVED } \\
\text { SPECTRAL INDEX } \\
\alpha(1400-57.5) \\
(6)\end{array}$} & \multirow[b]{2}{*}{$\begin{array}{c}S_{\text {cen }} / S_{\text {tot }} \\
(1400 \mathrm{MHz}) \\
(7)\end{array}$} \\
\hline & $\begin{array}{c}S_{1400} \\
(\mathrm{mJy}) \\
(2)\end{array}$ & $\begin{array}{c}\alpha \\
(\mathrm{mJy}) \\
(3)\end{array}$ & $\begin{array}{c}S_{5000} \\
(4)\end{array}$ & & & \\
\hline NGC $253 \ldots$ & 6000 & -0.72 & 2400 & $60 \pm 10$ & $\ldots$ & 0.45 \\
\hline NGC $628 \ldots \ldots \ldots \ldots \ldots \ldots$ & 190 & -0.73 & 75 & $2 \pm 0.8$ & $\ldots$ & $<0.02$ \\
\hline NGC $891 \ldots \ldots \ldots \ldots \ldots$ & 770 & -0.85 & 260 & $11.7 \pm 2.0$ & $\ldots$ & 0.04 \\
\hline NGC $1055 \ldots \ldots \ldots \ldots \ldots \ldots$ & 180 & $\ldots$ & $\ldots$ & $\ldots$ & -0.63 & $<0.11$ \\
\hline NGC $1068 \ldots$ & 5000 & -0.79 & 1830 & $63 \pm 10$ & $\ldots$ & 0.80 \\
\hline IC $342 \ldots \ldots \ldots$ & 2250 & -0.95 & 670 & $45 \pm 20$ & $\ldots$ & 0.06 \\
\hline NGC $1569 \ldots \ldots \ldots \ldots \ldots \ldots$ & 430 & -0.45 & 240 & $1.5 \pm 0.5$ & $\ldots$ & $<0.06$ \\
\hline NGC $1961 \ldots$ & 170 & $\ldots$ & $\ldots$ & $\ldots$ & -0.77 & $<0.06$ \\
\hline NGC $2146 \ldots$ & 1080 & -0.63 & 485 & $8.1 \pm 2.0$ & $\ldots$ & 0.23 \\
\hline NGC $2805 \ldots \ldots \ldots \ldots \ldots \ldots$ & $<50$ & $\ldots$ & $\ldots$ & $\ldots$ & $<-0.8$ & $\ldots$ \\
\hline NGC $2814 \ldots \ldots \ldots \ldots \ldots \ldots$ & 20: & $\ldots$ & $\ldots$ & $\ldots$ & -0.72 : & $\ldots$ \\
\hline NGC $2841 \ldots \ldots \ldots \ldots \ldots$ & 75 & -0.78 & 28 & $0.9 \pm 0.2$ & $\ldots$ & $<0.13$ \\
\hline NGC $2903 \ldots$. & 450 & -0.74 & 175 & $4.8 \pm 1.0$ & $\ldots$ & 0.17 \\
\hline NGC $2964 \ldots \ldots \ldots \ldots \ldots \ldots$ & 100 & -0.88 & 33 & $1.7 \pm 0.4$ & $\ldots$ & 0.38 \\
\hline NGC $3031 \ldots \ldots \ldots \ldots \ldots$ & 450 & -0.62 & 205 & $3.2 \pm 0.5$ & $\ldots$ & 0.13 \\
\hline NGC $3034 \ldots \ldots \ldots \ldots \ldots$ & 10000 & -0.27 & 7100 & $23.8 \pm 5.0$ & $\ldots$ & 0.06 \\
\hline NGC 3079 ............... & 850 & -0.73 & 335 & $8.8 \pm 1.0$ & $\ldots$ & 0.19 \\
\hline NGC $3221 \ldots \ldots \ldots \ldots \ldots$ & 170: & $\ldots$ & $\ldots$ & $\ldots$ & $-0.2:$ & $\ldots$ \\
\hline NGC $3227 / 6 \ldots \ldots \ldots \ldots \ldots$ & 100 & -0.66 & 430 & $0.8 \pm 0.2$ & $\ldots$ & 0.95 \\
\hline NGC $3310 \ldots \ldots \ldots \ldots \ldots$ & 380 & -0.69 & 160 & $3.5 \pm 0.7$ & $\ldots$ & $<0.03$ \\
\hline NGC 3351 .............. & 75 & -0.69 : & 31: & 0.7: & $\ldots$ & 0.40 \\
\hline NGC $3368 \ldots \ldots \ldots \ldots \ldots$ & 90 & $\ldots$ & $\ldots$ & $\ldots$ & $>-0.4$ & $<0.11$ \\
\hline NGC $3521 \ldots \ldots \ldots \ldots \ldots$ & 430 & $\ldots$ & $\ldots$ & $\ldots$ & -1.1 & $<0.05$ \\
\hline NGC $3556 \ldots \ldots \ldots \ldots \ldots$ & 295 & -0.85 & 100 & $4.5 \pm 0.8$ & $\ldots$ & 0.04 \\
\hline NGC $3627 \ldots \ldots \ldots \ldots \ldots$ & 450 & -0.80 & 165 & $5.8 \pm 1.0$ & $\ldots$ & $<0.04$ \\
\hline NGC $3628 \ldots \ldots \ldots \ldots \ldots \ldots$ & 510 & -0.64 & 225 & $4.0 \pm 0.9$ & $\ldots$ & 0.43 \\
\hline NGC $4027 \ldots$ & 115 & $\ldots$ & $\ldots$ & $\ldots$ & -0.76 & $<0.17$ \\
\hline NGC 4038/9 $\ldots \ldots \ldots \ldots \ldots$ & 520 & -0.7 & 215 & $4.9 \pm 1.5$ & $\ldots$ & $<0.04$ \\
\hline NGC $4085 \ldots \ldots \ldots \ldots \ldots$ & 50 & $\ldots$ & $\ldots$ & $\ldots$ & $>-0.55$ & $\ldots$ \\
\hline NGC $4088 \ldots \ldots \ldots \ldots \ldots$ & 200 & -0.78 & 76 & $2.5 \pm 0.5$ & $\ldots$ & $<0.10$ \\
\hline NGC $4102 \ldots \ldots \ldots \ldots \ldots$ & 300 & -0.7 & 125 & $2.5 \pm 0.6$ & $\cdots$ & 0.67 \\
\hline NGC $4157 \ldots \ldots \ldots \ldots \ldots \ldots$ & 200 & -0.72 & 80 & $2.0 \pm 0.6$ & $\ldots$ & $<0.10$ \\
\hline NGC 4217 ............... & 115 & $\ldots$ & $\ldots$ & $\ldots$ & $-0.3:$ & $<0.17$ \\
\hline NGC $4254 \ldots \ldots \ldots \ldots \ldots$ & 530 & -0.77 & 200 & $6.2 \pm 1.0$ & $\ldots$ & $<0.04$ \\
\hline NGC $4258 \ldots \ldots \ldots \ldots \ldots$ & 820 & -0.78 & 305 & $10.0 \pm 1.0$ & $\ldots$ & $<0.01$ \\
\hline NGC $4302 \ldots \ldots \ldots \ldots \ldots$ & 43 & $\ldots$ & $\ldots$ & $\ldots$ & $>-0.6$ & $\ldots$ \\
\hline NGC $4321 \ldots \ldots \ldots \ldots \ldots$ & 300 & -0.85 & 100 & $4.6 \pm 1.0$ & $\ldots$ & 0.17 \\
\hline NGC 4490/85 ............ & 850 & -0.75 & 325 & $9.0 \pm 2.0$ & $\ldots$ & 0.02 \\
\hline NGC 4631/27 $\ldots \ldots \ldots \ldots \ldots$ & 1300 & -0.73 & 515 & $13.5 \pm 4.0$ & $\cdots$ & 0.04 \\
\hline NGC 4656/7 $\ldots \ldots \ldots \ldots \ldots$ & 120 & -0.46 & 67 & $0.5 \pm 0.1$ & $\ldots$ & $<0.08$ \\
\hline NGC 4736 .............. & 270 & -0.61 & 125 & $1.9 \pm 0.2$ & $\ldots$ & 0.10 \\
\hline NGC 5005 .............. & 165 & -0.9 & 53 & $2.9 \pm 0.5$ & $\ldots$ & 0.52 \\
\hline NGC $5033 \ldots \ldots \ldots \ldots \ldots$ & 270 & -0.73 & 105 & $2.8 \pm 0.4$ & $\ldots$ & 0.22 \\
\hline NGC 5055 .............. & 409 & -0.78 & 150 & $5.0 \pm 0.6$ & $\ldots$ & $<0.02$ \\
\hline NGC 5169/73 ............ & 120 & $\ldots$ & $\ldots$ & $\ldots$ & $>-0.03$ & $\ldots$ \\
\hline NGC 5194/5 $\ldots \ldots \ldots \ldots \ldots$ & 1500 & -0.8 & 540 & $20.0 \pm 4.0$ & $\ldots$ & 0.05 \\
\hline NGC 5236 ............. & 2275 & -0.74 & 950 & $25.0 \pm 2.0$ & $\ldots$ & 0.14 \\
\hline NGC $5457 \ldots \ldots \ldots \ldots \ldots$ & 800 & $-0.7:$ & 330: & $7.5 \pm 2.5$ & $\ldots$ & $<0.01$ \\
\hline 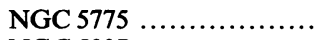 & 220: & $-0.8:$ & 80: & 2.9: & $\ldots$ & $\ldots$ \\
\hline NGC 5907 ............... & 200 & -0.79 & 73 & $2.5 \pm 0.5$ & $\ldots$ & $<0.05$ \\
\hline NGC $6946 \ldots \ldots \ldots \ldots \ldots$ & 1550 & -0.7 & 635 & $14.5 \pm 2.0$ & $\ldots$ & 0.06 \\
\hline NGC 7317-20 ............ & 100: & $\ldots$ & $\ldots$ & $\ldots$ & $-0.7:$ & $\ldots$ \\
\hline NGC $7331 \ldots \ldots \ldots \ldots \ldots$ & 486 & -0.9 & 155 & $8.7 \pm 1.4$ & $\cdots$ & $<0.02$ \\
\hline
\end{tabular}

NoTE.-Semicolon indicates uncertain values.

$\mathrm{GHz}$ flux density that we could determine from the literature. Column (3) gives the best value for the spectral index $\alpha$ in the high frequency range (usually 1-5 GHz). Columns (4) and (5) give flux densities at $5 \mathrm{GHz}$ and $57.5 \mathrm{MHz}$, respectively, determined from the values in columns (2) and (3). In some cases, the existing information is insufficent to derive a spectal index, although a reasonably accurate $1.4 \mathrm{GHz}$ flux density value exists. For those cases, we give in column (6) the observed spectral index between $57.5 \mathrm{MHz}$ and $1.4 \mathrm{GHz}$. As we will show below, this observed spectral index generally underestimates the steepness of the spectrum at higher frequencies. Finally, column (7) gives the fraction of the total $1.4 \mathrm{GHz}$ emission associated with the central region of the galaxy $\left(S_{\text {cen }} / S_{\text {tot }}\right)$ based on the survey by Hummel (1980).

Nineteen galaxies detected at $57.5 \mathrm{MHz}$ are not included in comparison (Table 5) because they lack reliable high-frequency data. Of the 53 galaxies in Table 5, 11 have only limited highfrequency data and four (NGC 3368, 4085, 4302, and 5169/73) 

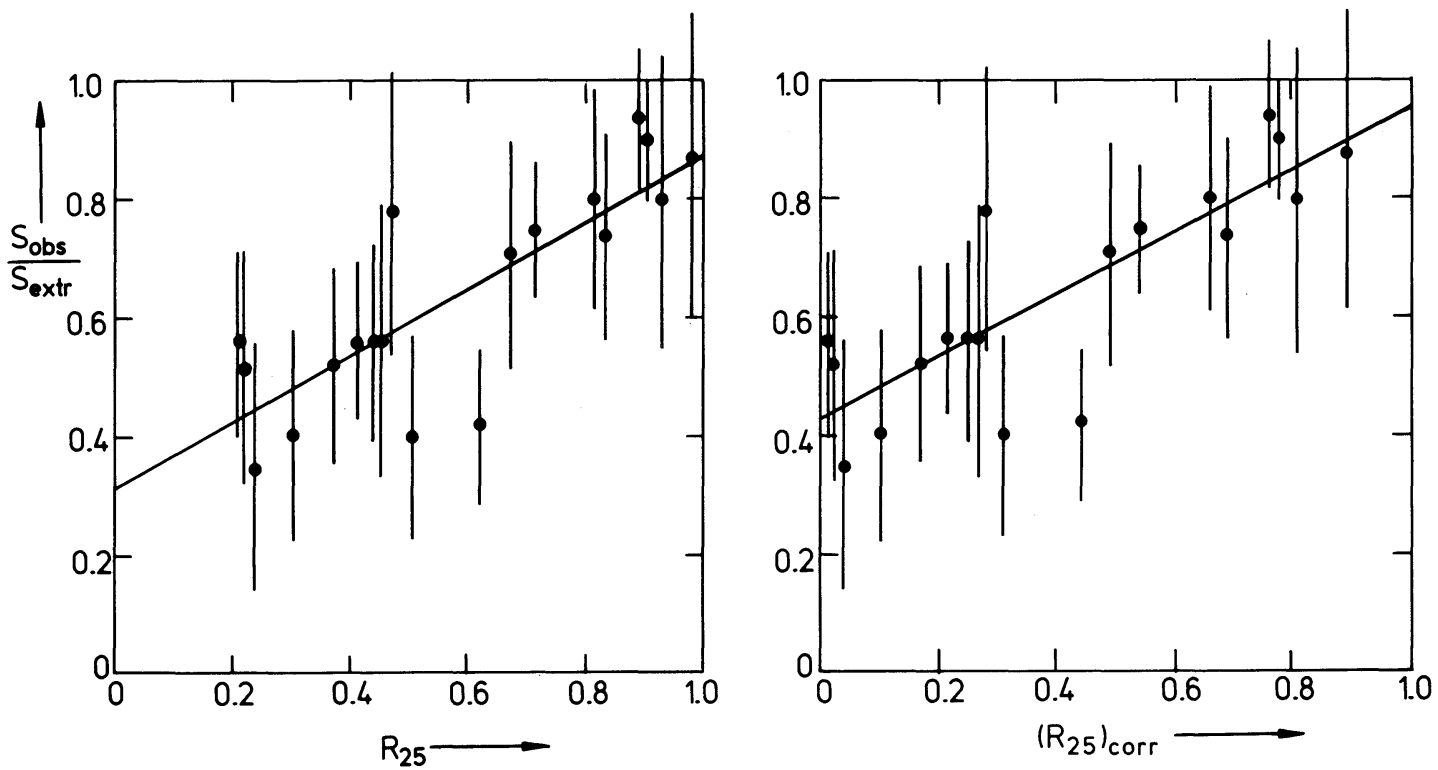

Fig. 2. - (a) Ratio of observed to extrapolated flux densities at $57.5 \mathrm{MHz}$ as a function of galaxy axial ratio $R_{25}$ for disk-dominated galaxies. Straight line indicates linear regression fit to all data points. (b) Same as $(a)$, but axial ratio $R_{25}$ corrected for finite disk thickness of 0.2 disk diameter, i.e., the maximum width to diameter ratio consistent with edge-on galaxies in the sample.

were not detected at $57.5 \mathrm{MHz}$. Since these four galaxies are among the weakest sources at $1.4 \mathrm{GHz}$, it is reasonable to assume that the majority of galaxies with only upper limits at $57.5 \mathrm{MHz}$ are in fact weak emitters at all frequencies. Of the remaining 38 galaxies in Table 5, 21 are disk-dominated spirals $\left(S_{\text {cen }} / S_{\text {tot }}<0.1\right.$ at $\left.1.4 \mathrm{GHz}\right), 14$ are spirals with significant central emission $\left(S_{\text {cen }} / S_{\text {tot }}>0.1\right.$ at $\left.1.4 \mathrm{GHz}\right)$, and three are irregulars (NGC 1569, 3034, 4038/9). A straightforward comparison of the observed and extrapolated $57.5 \mathrm{MHz}$ fluxdensities of these 38 galaxies shows that on average, the observed values $S_{\text {obs }}$ are significantly below the extrapolated values $S_{\text {extr }}$. At least qualitatively, we therefore confirm the

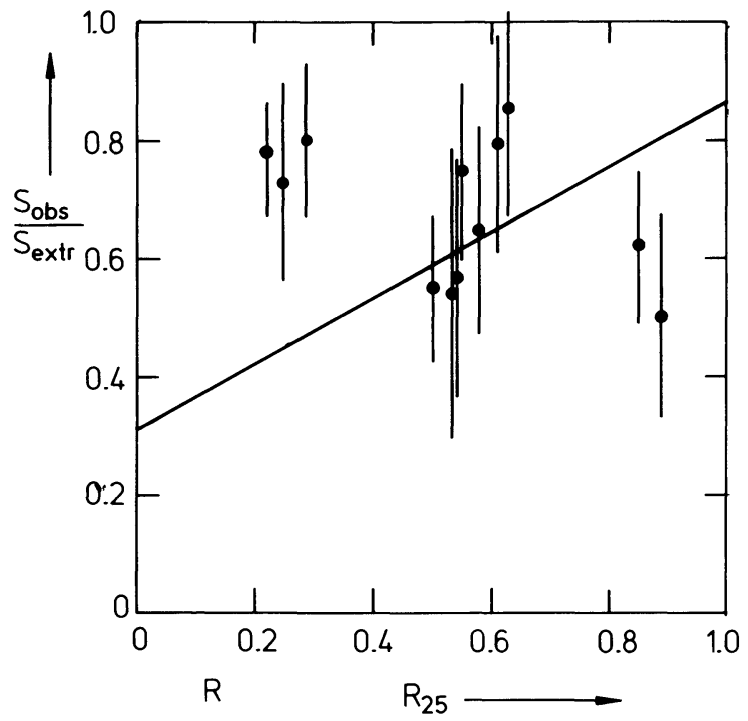

Fig. 3.-Same as Fig. $2 a$, but for galaxies with strong central component $\left(S_{\text {cen }} / S_{\text {tot }}>0.1\right.$ at $\left.1400 \mathrm{MHz}\right)$. The linear regression fit from Fig. $2 a$ is added for comparison. conclusion of Slee $(1972 b)$ that galaxy spectra tend to flatten at low frequencies.

For a further analysis of this result, we plotted the ratio $S_{\text {obs }} / S_{\text {extr }}(57.5)$ as a function of axial ratio $R_{25}$, the ratio of minor to major axes at a surface brightness in $B$ of 25 mag $\operatorname{arcsec}^{-2}$ as given by de Vaucouleurs, de Vaucouleurs, and Corwin (1976). Furthermore, we separated the disk-dominated galaxies from those with significant central emission. For the latter, the derivation of extrapolated flux densities is not straightforward, because disks and central regions may have rather different radio spectra (e.g., M81, see de Bruyn et al. 1976; Emerson, Kronberg, and Wielebinski 1978). The resulting plots are shown in Figures $2 a$ (disk-dominated) and 3 (significant central emission). For galaxies with infinitely thin disks, the axial ratio is directly related to the galaxy tilt $\left(R_{25}=\right.$ sin i). Galaxies with finite disk thickness need a correction. Hence, in Figure $2 b$ we have assumed a disk thickness-todiameter ratio of 0.2 which is the highest ratio consistent with Figure $2 a$. This yields corrected axial ratios $\left(R_{25}\right)_{\text {corr }}$. Note that in Figures $2 a$ and $2 b$, three galaxies are excluded: NGC 4656 has a poor signal-to-noise ratio, IC 342 is large compared to our beam, and NGC 5907 is confused with a background source at its southern tip (Hummel 1980).

Disk-dominated galaxies show a clear correlation between $S_{\text {obs }} / S_{\text {extr }}$ and $R_{25}:$ in Figure $2 a, S_{\text {obs }} / S_{\text {extr }}(57.5)=0.56\left(R_{25}\right)$ +0.31 , while in Figure $2 b, S_{\text {obs }} / S_{\text {extr }}(57.5)=0.52\left(R_{25}\right)_{\text {corr }}$ +0.43 (both with correlation coefficient $r=0.82$ for $n=18$ ). This result implies that on average, $87 \%-95 \%$ of the extrapolated flux density is actually seen in face-on galaxies, whereas for edge-on galaxies, only about $31 \%-43 \%$ of the expected flux density is seen. The significance of this correlation is illustrated by a comparison with Figure 3 . The sample of galaxies with central emission shows, if anything, an anticorrelation. We will return to that result and its relevance for the properties of core emission in galaxies in a later paper.

One further remark is in order. The extrapolated $57.5 \mathrm{MHz}$ flux densities in Table 5, used in Figures $2 a$ and $2 b$, were deter- 
mined by using spectral index information derived from observations in the $1.4-5 \mathrm{GHz}$ range. Consequently, they are systematically underestimated if significant thermal emission is present at these frequencies. This results in a systematic overestimate of the ratio $S_{\text {obs }} / S_{\text {extr }}$ in Figures $2 a$ and $2 b$. We illustrate this as follows. The mean spectral index for the sample shown in Figures $2 a$ and $2 b$ is $\alpha=-0.75$. If the thermal fraction at $5 \mathrm{GHz}\left(F_{5}\right)$ is as high as $50 \%$ (upper limit from Paper I), a spectral index of -0.1 for optically thin thermal emission then implies an intrinsic nonthermal spectral index $\alpha_{\mathrm{NT}}=$ -1.1 and a correction factor to $S_{\text {obs }} / S_{\text {extr }}$ of 0.40 , increasing the discrepancy between observed and extrapolated flux densities by more than a factor of 2 . At the same time, note that thermal processes do not contribute significantly to the emission at $\mathbf{5 7 . 5}$ $\mathrm{MHz}$ : for $F_{5}=0.5$, thermal contributions at $57.5 \mathrm{MHz}$ are only about $1 \%$. In the following, $\left(S_{\text {obs }} / S_{\text {extr }}\right)_{\text {corr }}$ indicates the ratio between observed and extrapolated flux densities corrected for the effect of thermal emission at high frequencies; the magnitude of the correction depends on the assumed value of $F_{5}$.

\section{b) Free-Free Absorption in Spiral Galaxies}

What could cause disk-dominated galaxies to have observed flux densities systematically lower than the extrapolated flux densities? In principle, several mechanisms may be responsible for a low-frequency turnover of nonthermal radio continuum spectra: (a) synchrotron self-absorption, (b) the RazinTsytovich effect, $(c)$ cyclotron turnovers, $(d)$ low-energy cutoffs in the electron injection spectrum, and $(e)$ free-free absorption by thermal plasmas. With the exception of free-free absorption, none of these mechanisms appear applicable to a turnover at frequencies near $57.5 \mathrm{MHz}$. For instance, the cutoff frequency for mechanism (a) (synchrotron self-absorption) is $v_{t}=34$ $\left(S_{\max } / \theta^{2}\right)^{0.4} B^{0.2} \mathrm{MHz}$ (Kellermann and Pauliny-Toth 1969), where the flux density $S_{\max }$ is in janskys, the source size $\theta$ is in arcseconds, and the magnetic field $B$ is in G. Since the galaxies in our sample are characterized by surface brightnesses $\left(S_{\max } / \theta^{2}\right)^{0.4}=4 \times 10^{-2}$ or less, a turnover near $57.5 \mathrm{MHz}$ could only take place for unreasonably high magnetic field strengths of order $0.5 \mathrm{G}$ (Kellermann and Pauliny-Toth 1969). In a similar way, for more reasonable magnetic field strengths of order $10^{-6}-10^{-5} \mathrm{G}$, a spectral turnover due to mechanisms $(b),(c)$, and $(d)$ also takes place at frequencies far below 50 MHz. Hence, we conclude that mechanism (e) (free-free absorption) is the only viable explanation for the observed turnover.

The dependence of absorption on axial tilt observed for the sample of disk-dominated spiral galaxies suggests that a significant fraction of the nonthermal emission of these galaxies is absorbed by ionized material in their disks. The relatively small emerging fraction for edge-on galaxies (Figs. $2 a, 2 b$ ) indicates that on average, the radial extent and scale height of the absorbing thermal plasma must be comparable to the extent and scale height of the nonthermal plasma, and that nonthermal halos are not the major contributor to the emission at very low frequencies.

We note with interest the ratio $S_{\text {obs }} / S_{\text {extr }}=0.56 \pm 0.15$ obtained for the edge-on galaxy NGC 891. This ratio indicates a maximum nonthermal halo or "thick disk" contribution of $43 \% \pm 7 \%$ at $57.5 \mathrm{MHz}$, consistent with $0.6-5.0 \mathrm{GHz}$ aperture synthesis observations that suggest a $40 \%$ contribution at 5 $\mathrm{GHz}$ by a nonthermal "thick disk" (Allen, Baldwin, and Sancisi 1978). This example also shows that part of the spread in Figures $2 a$ and $2 b$ may be intrinsic and due to differences in thick disk contribution. If "thick disk" emission is common (see Harnett and Reynolds 1985; Beuermann, Kanbach, and Berkhuysen 1985), a possible contribution by nonthermal halo components becomes so small as to be negligible.

In the following, we explore the general consequences of these conclusions by applying two simple models (see Appendix) to the "mean" galaxy in Figure $2 b$, which represents the mildest case of free-free absorption. Model 1 assumes absorption of nonthermal emission by individual $\mathrm{H}$ II regions covering only a fraction of the face-on disk, while model 2 assumes absorption to take place homogeneously in a thermal cylinder embedded in a larger nonthermal cylinder. Part of the nonthermal emission escapes the galaxy without having suffered absorption; an upper limit to the fraction of this emission is given by the value of $\left(S_{\text {obs }} / S_{\text {extr }}\right)_{\text {corr }}$ at $\left(R_{25}\right)_{\text {corr }}=0$.

The models enable us to determine the $57.5 \mathrm{MHz}$ optical depth $\tau_{\text {obs }}$ of the absorbing thermal plasma in the "mean" galaxy from $\left(S_{\mathrm{obs}} / S_{\text {extr }}\right)_{\text {corr }}$. The optical depth $\tau$ at a frequency $v$ is related to the emission measure EM of the absorbing ionized gas by (Oster 1961; see also Gayet 1970):

$$
\tau_{v}=3.014 \times 10^{-2} T_{\mathrm{e}}^{-1.5} v^{-2} \operatorname{EM} f\left(v, T_{e}\right),
$$

in which:

$$
f\left(v, T_{e}\right)=\ln \left(4.955 \times 10^{-2} v^{-1}\right)+1.5 \ln T_{e} ;
$$

$T_{e}$ is in $\mathrm{K}, v$ is in gigahertz, and EM is in $\mathrm{pc} \mathrm{cm}^{-6}$. From this, we derive at a frequency of $57.5 \mathrm{MHz}$ the relation $\tau_{\mathrm{obs}}=1.1$ $\times 10^{2} T_{e}^{-1.5} \mathrm{EM}$. In view of the results presented below, we have assumed a $T_{e}=3000 \mathrm{~K}$. In any case, the dependence of $f\left(v, T_{e}\right)$ on $T_{e}$ is weak. Moreover, at $5 \mathrm{GHz}$, thermal emission is given by:

$$
S_{5, T} \theta^{-2}=0.143 T_{e}^{-0.35} \mathrm{EM},
$$

with $S_{5, T}$ in millijanskys and $\theta$ in arcminutes (e.g., Mezger and Henderson 1967). Thus, if the high-frequency thermal emission and the low-frequency thermal absorption are known, the electron temperature $T_{e}$ of the thermal plasma can be determined. In the following, we find it convenient to use the thermal emission fraction at $5 \mathrm{GHz}\left(F_{5}\right)$ as a measure for the high-frequency thermal emission. We define for the face-on disk $\theta=2 r_{T}=2 a$ $r_{\mathrm{NT}}$, and $r_{\mathrm{NT}}=0.5 f D_{25}$. The filling factors $a$ and $f$ relate thermal radio extent to nonthermal radio extent and nonthermal radio extent to optical extent, respectively (see Appendix). For the galaxies making up the sample shown in Figure 1, the quantity $S_{5, \text { tot }} D_{25}^{-2}=4.0 \pm 0.9 \mathrm{mJy} \mathrm{arcmin}^{-2}$ (from $S_{5, \text { tot }}$ as given in Table 5 and $D_{25}$ by de Vaucouleurs, de Vaucouleurs, and Corwin 1976). Thus,

$$
F_{5}=S_{5, T} / S_{5, \text { tot }}=3.6 \times 10^{-2} a^{2} f^{2} T_{e}^{-0.35} \mathrm{EM} .
$$

\section{c) Absorption by Discrete $\mathrm{H}$ II Regions}

Can the free-free absorption be caused by discrete $\mathrm{H}$ II regions in the observed galaxies? Such $\mathrm{H}$ II regions have emission measures typically $\mathrm{EM} \approx 3 \times 10^{3} \mathrm{pc} \mathrm{cm}^{-6}$ (see M33, M101; Israel and Van der Kruit 1973; Israel, Goss, and Allen 1975) and electron temperatures $T_{e} \approx 0.8 \times 10^{4} \mathrm{~K}$ (Smith 1975; Shields and Searle 1978; Hawley 1978; Kwitter and Aller 1981), and cover at most a few percent of the face-on galactic disk (see Hodge and Kennicutt 1983); thus, we take the surface filling factor $a^{\prime 2} \leq 0.05$. The high electron temperatures and 
small filling factors of such $\mathrm{H}$ il regions lead us to believe that they cannot explain the free-free absorption implied by our observations. This is borne out by numerical estimates. With the above values for $T_{e}$ and EM, a typical $\mathrm{H}$ in region has an optical depth $\tau_{\text {obs }}=0.5$. The ratio $\left(S_{\text {obs }} / S_{\text {extr }}\right)_{\text {corr }}<0.95$ for $F_{5}>0$ (§ III $\left.a\right)$ implies $a^{\prime 2}>0.25$ (see Appendix, model 1), so that a significant fraction of the galactic disk has to be covered by such $\mathrm{H}$ II regions. Very few galaxies indeed show $\mathrm{H} \alpha$ images consistent with such a high fraction (see, e.g., Hodge and Kennicutt 1983). Even worse, the above values for $T_{e}$ and EM imply $F_{5}=4.7 a^{2} f^{2}=4.7 a^{\prime 2}$. Since $F_{5}<0.5$ (see Paper I), we require $a^{\prime 2}<0.11$, which is in clear contradiction to the value found above. In fact, there is no reasonable combination of $T_{e}$ and EM that simultaneously satisfies $F_{5}<0.5, a^{\prime 2}<0.05$, and implied values of $\left(S_{\text {obs }} / S_{\text {extr }}\right)_{\text {corr }}$. We conclude that the free-free absorption indicated by Figure $2 b$ cannot be caused by discrete $\mathbf{H}$ II regions of the kind seen in late-type galaxies such as M33 and M101.

Rather, the results of model 1 for $F_{5}>0$ point to ionized regions filling most of the nonthermal emission region and having low electron temperatures and low emission measures, as the agent responsible for free-free absorption. This situation is more accurately described by model 2 .

\section{d) Absorption by an Extended, Diffuse Ionized Gas}

Combining equations (1) and (3), we find for the electron temperature $T_{e}$ of the absorbing plasma:

$$
T_{e}^{1.15}=3.1 \times 10^{3}\left[F_{5} /\left(a^{2} \tau_{\text {obs }}\right)\right] f^{-2} .
$$

For the face-on galaxy in Figure $2 b$, we have calculated the quantity $F_{5} /\left(a^{2} \tau_{\text {obs }}\right)$ predicted by model 2 for a few representative values of $F_{5}, a$, and $b ; b=h_{T} / h_{\mathrm{NT}}$ is the ratio between thermal and nonthermal disk thickness. Figure 4 shows a maximum of 0.35 for $a=1, b=1$ (thermal and nonthermal volumes, coincide) and $F_{5}=0.20$. In the model, the ratio of (cylindrical) disk thickness to diameter is $h_{\mathrm{NT}} / 2 r_{\mathrm{NT}}=0.64(a / b)$ $\left(\tau_{\text {face-on }} / \tau_{\text {edge-on }}\right)$; see Appendix. In Figure 5, we show this ratio

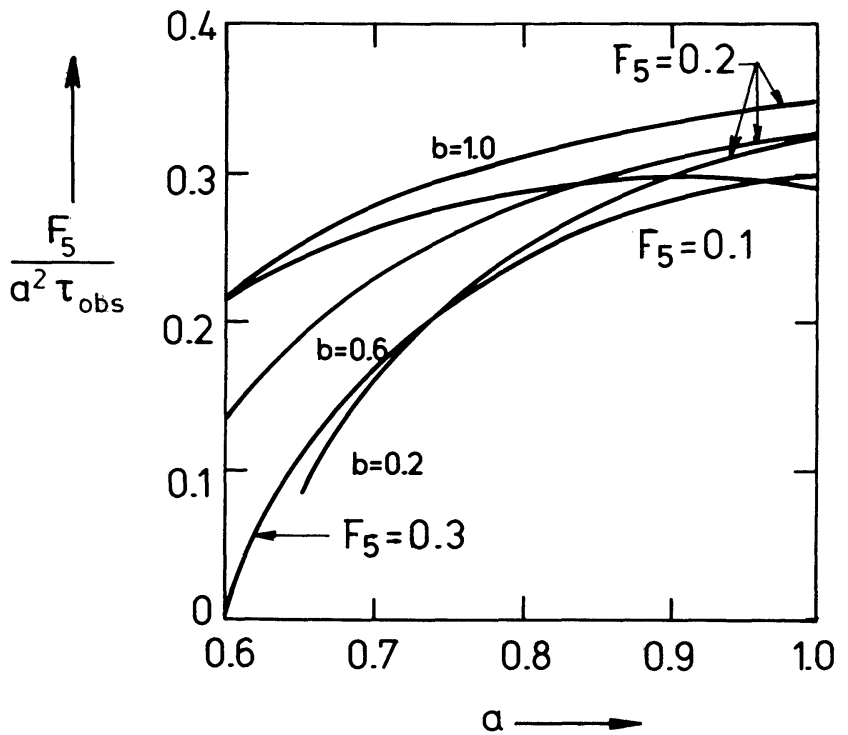

Fig. 4.-Variation of the quantity $F_{5} /\left(a^{2} \tau_{\mathrm{obs}}\right)$ in model 2 as a function of model scale parameter $a$, for a variety of values $F_{5}$ (see text for explanation); $b=1.0$. In order to illustrate the effect of model scale parameter $b$, we have added curves for $b=0.6$ and $b=0.2$ in the case $F_{5}=0.2$. as a function of $a$ and $b$ for $F_{5}=0.1,0.2$, and 0.3. The limiting case $\tau_{\text {edge-on }}=\infty$ implies that we must have $b>1$ $-\left(S_{\text {obs }} / S_{\text {extr }}\right)_{\text {corr }}$ for all values of $a$. We can constrain the filling factors $a$ and $b$ by requiring a minimum thickness for the nonthermal radio disk. An "infinitely thin" disk with $b=1$ would still have a volume filling factor $a^{2} b \geq 50 \%$; for a disk thickness diameter ratio of 0.1 , we find a volume filling factor $a^{2} b$ of at least $65 \%$, while for a ratio of 0.15 the volume filling factor is between $75 \%$ and unity. Since a thickness diameter ratio greater than 0.1 seems applicable (see Fig. 2), we conclude to a lower limit of $65 \%$ to the volume filling factor of the absorbing thermal gas with respect to the nonthermal volume. At this point, we wish to emphasize that, whatever the actual distribution of nonthermal and thermal plasma, the absorbing material has to fill a large fraction of the nonthermal emitting volume, as is in fact illustrated by the relatively small value of $\left(S_{\text {obs }} / S_{\text {extr }}\right)_{\text {corr }}$ of edge-on galaxies.

Since the quantity $F_{5} / a^{2} \tau_{\text {obs }}<0.35$ for all values $a, b<1$ (see Fig. 4), equation (4) yields an upper limit to the electron temperature of the absorbing gas $T_{e}<435 f^{-1.74}$. Observational uncertainties correspond to a possible error of about $30 \%$. Out of the 19 galaxies in the sample, 14 have aperture synthesis radio maps from which the factor $f$ (radio diameter/ optical diameter) may be estimated. We find a lower limit $f>0.5$, and a mean value $f \approx 0.8$. We thus obtain rather low electron temperatures $T_{e}<1450 \mathrm{~K}$ and more probably $T_{e}<$ $650 \mathrm{~K}$. These limits change little for $0.1<F_{5}<0.3$. Inserting these limits on $T_{e}$ into equation (1), we also obtain limits on the face-on disk emission measure of roughly $\mathrm{EM}<1500 F_{5}$. In models more realistic than our crude model 2 , such as exponential disks, both the thermal and the nonthermal gas will be concentrated toward the inner parts, resulting in a smaller effective value of $f$. However, even with a factor $f$ as small as 0.3 , we still have $T_{e}<3500 \mathrm{~K}$, while the face-on emission measure $\mathrm{EM}<5600 F_{5}$. We emphasize that all these values are true upper limits. For instance, if $a, b<1$, free-free absorption has to be more efficient to yield the same observational result, so that the limit on $T_{e}$ is lowered even further. Likewise, a (probably large) part of the thermal emission at $5 \mathrm{GHz}$ is caused by discrete $H$ II regions (see Israel 1980) lowering the effective value of $F_{5}$ in equations (3) and (4), and thereby again decreasing permittable values of $T_{e}$.

There are further constraints on $T_{e}$. H $\alpha$ observations of latetype galaxies suggest emission measures of $30 \mathrm{pc} \mathrm{cm}^{-6}$ or less if $T_{e}=7500 \mathrm{~K}$ (Deharveng and Pellet 1970; Sivan 1974; Monnet 1974; Torres-Peimbert, Lezcano-Araujo, and Peimbert 1974). For constant $\mathrm{H} \alpha$ intensity, EM is roughly proportional to $T_{e}^{0.8}$ (see Martin 1988), so that we require $T_{e}^{-0.81} \mathrm{EM} \leq 0.02$. Thus, with equation (1), $T_{e}<3.6 \tau_{\mathrm{obs}}^{-1.45}$. For $\tau_{\mathrm{obs}}=0.10\left(F_{5} \approx 0\right)$, $T_{e}<100 \mathrm{~K}$ and $\mathrm{EM}<1 \mathrm{pc} \mathrm{cm}^{-6}$. This upper limit decreases rapidly for smaller EM and larger fractions $F_{5}$. A similarly stringent constraint follows from power considerations. To maintain the ionization of the thermally absorbing gas, we need a power input $P=\alpha n_{e}^{2} V E_{i}$ ergs $s^{-1}$, in which the effective recombination coefficient $\alpha=2 \times 10^{-10} T_{e}^{-0.71} \mathrm{~cm}^{3} \mathrm{~s}^{-1}$ for $T_{e} \approx 10^{3} \mathrm{~K}$ (accurate to about $10 \%$ for the range $10^{2}<T_{e}<$ $10^{4} \mathrm{~K}$; see Martin 1988), $n_{e}$ is the electron density, $V$ is the total volume of thermal material, and $E_{i}$ is the ionization potential. Considering that the mean optical radius of the galaxies in the sample is $15 \mathrm{kpc}$, and expressing $P$ in units of $10^{44} \mathrm{ergs} \mathrm{s}^{-1}$, we rewrite this to

$$
P_{44}=0.905 f^{2} \mathrm{EM} T_{e}^{-0.71}
$$



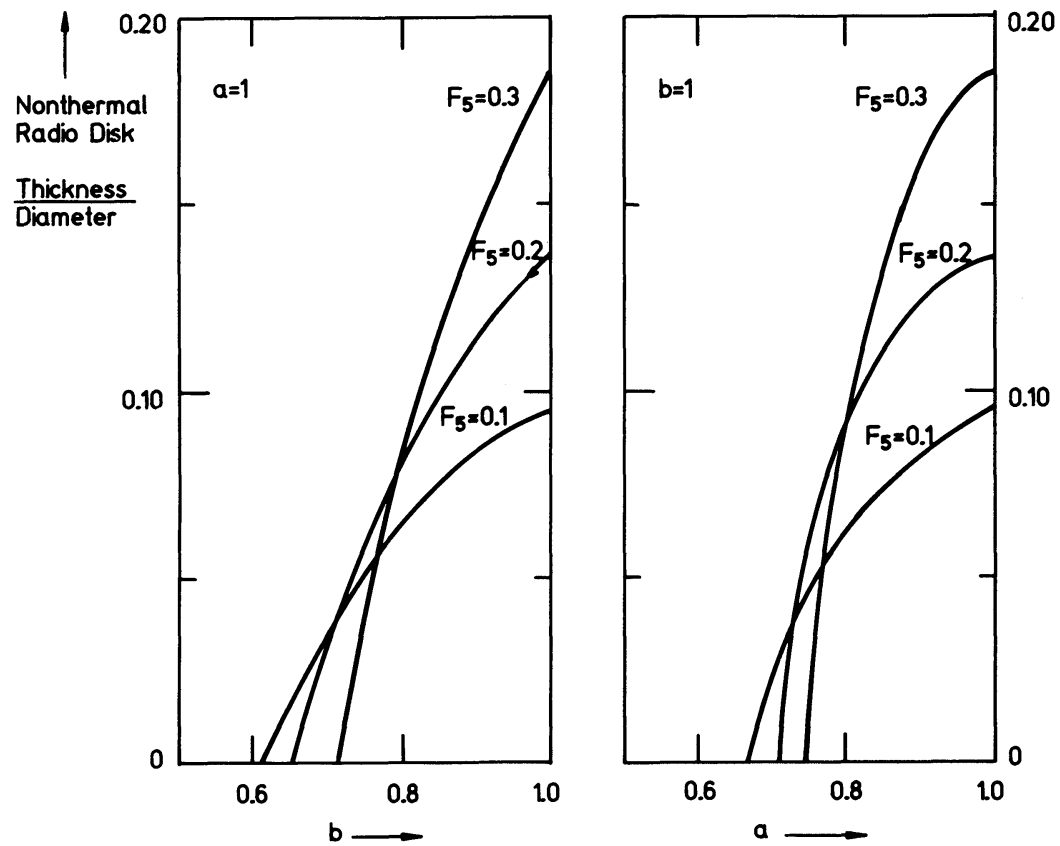

Fig. 5.-Nonthermal disk thickness/disk diameter ratio as a function of filling factors $a$ and $b$, for representative values of thermal fraction $F_{5}$

Combining equations (1) and (5), we find $T_{e}^{0.79}=122$ $P_{44} \tau_{\text {obs }}^{-1} f^{-2}$. The main source of power injected into the interstellar medium of disk-dominated galaxies is the ensemble of early-type stars; the total (hydrogen ionizing) power injected into the Galaxy is about $P_{44}=0.04$ (Cruz-Gonzalez et al. 1974; Panagia and Terzian 1984; Bregman and Harrington 1986). About one-half to two-thirds of this amount is absorbed by discrete "classical" $\mathrm{H}$ II regions (Cruz-Gonzalez et al. 1974; Bregman and Harrington 1986; Kulkarni and Heiles 1988). If we take this result as representative for our sample, we have $P_{44}<0.04$ and $\tau_{\text {obs }}>0.1\left(F_{5} \approx 0\right)$, so that $T_{e}<135 f^{-2.4}$; thus, for $f=0.8$, we find $T_{e}<230 \mathrm{~K}$, while for larger values $F_{5}$ this upper limit again decreases rapidly.

We conclude that a smoothy distributed component filling a large fraction of the galaxy volume can only explain the observational results if $T_{e}$ is extremely low. By using equation (5) and assuming $T_{e}=50 \mathrm{~K}$, we find that such a component would have a density of order $n_{e}=0.01 \mathrm{~cm}^{-3}$ and a mass of a few times $10^{8} M_{\odot}$, i.e., roughly $5 \%$ of the Galactic $\mathrm{H}$ I mass. Its emission measure would be consistent with the observations. These values indicate that the absorbing thermal component most likely would be the ionized fraction of the cold neutral medium. Although this explanation appears feasible, we will now consider the effect of clumpiness as an alternative.

\section{e) Absorption by a Clumpy Ionized Gas}

High-resolution studies of the interstellar medium in the Galaxy show (often filamentary) structure on all scales, down to a fraction of a parsec (see, e.g., Schmidt Telescope Survey images; also Falgarone and Perault 1988). Most likely, there is a continuous distribution of clumps or filaments of varying size and density. However, both nonthermal emission and thermal absorption will preferentially arise from the most compressed (densest) volumes, We now make the simple assumption that both emission and absorption take place in identical clumps, with properties thus biased toward those of the densest condensations in the actual medium. We define $f_{c}^{3}$ as the volume fraction of the nonthermal disk filled with emitting material. Also, $F_{5}^{\prime}=f_{5} F_{5}$ is the fractional contribution of the free-free absorbing gas to the total emission at $5 \mathrm{GHz}$. Thus, we obtain from equations (3) and (5)

$$
F_{5}^{\prime}=3.6 \times 10^{-2} f^{2} f_{c}^{2} T_{e}^{-0.35} \mathrm{EM},
$$

and

$$
P_{44}=0.905 f^{2} f_{c}^{2} T_{e}^{-0.71} \mathrm{EM} .
$$

The optically observed emission measure $\mathrm{EM}_{0}=f_{c}^{2} \mathrm{EM}$. Here we limit ourselves to the case $F_{5}=0.2$, and we assume $f \approx 0.8$ as in $\S$ III $e$. Following the discussion in $\S$ III $c$, we also assume $a=b=1$ (as illustrated in Fig. 6, departures from this value have relatively little influence). The parameters of interest (clump electron temperature, clump emission measure, and surface filling factor) are then found from:

$$
\begin{gathered}
T_{e}=7760\left(F_{5}^{\prime} / P_{44}\right)^{2.78}, \\
\mathrm{EM}=6215 \tau_{\mathrm{obs}}\left(F_{5}^{\prime} / P_{44}\right)^{4.17}, \\
f_{c}^{2}=0.16 \tau_{\mathrm{obs}}^{-1}\left(F_{5}^{\prime} / P_{44}\right)^{-2.2} P_{44}
\end{gathered} .
$$

Average line-of-sight electron densities are given by

$$
\begin{aligned}
\left\langle n_{e}\right\rangle^{2} & =f_{c} \mathrm{EM} / h_{\mathrm{NT}} \\
& =1995 h_{\mathrm{NT}}^{-1} \tau_{\mathrm{obs}}^{1 / 2} P_{44}^{1 / 2}\left(F_{5}^{\prime} / P_{44}\right)^{3.07},
\end{aligned}
$$

while clump electron densities follow from

$$
n_{e}=1.25 f_{c}^{-1}\left\langle n_{e}\right\rangle .
$$

From equation (9), we may estimate the ratio $F_{5}^{\prime} / P_{44}$ as a function of nonthermal disk thickness $h_{\mathrm{NT}}$ and input power $P_{44}$ by taking the line-of-sight electron densities $\left\langle n_{e}\right\rangle$ found in the Galaxy from pulsar dispersion measures. Weisberg, Boriakoff, and Rankin (1979) suggest a mean value $\left\langle n_{e}\right\rangle \approx 0.03 \mathrm{~cm}^{-3}$; we also consider $\left\langle n_{e}\right\rangle=0.07$ and $0.10 \mathrm{~cm}^{-3}$, which may be more appropriate to inner parts of galaxies. A further constraint is imposed by the requirement $T_{e}^{-0.81} \mathrm{EM} \leq 0.02$ (see $\S$ III $d$ ). 


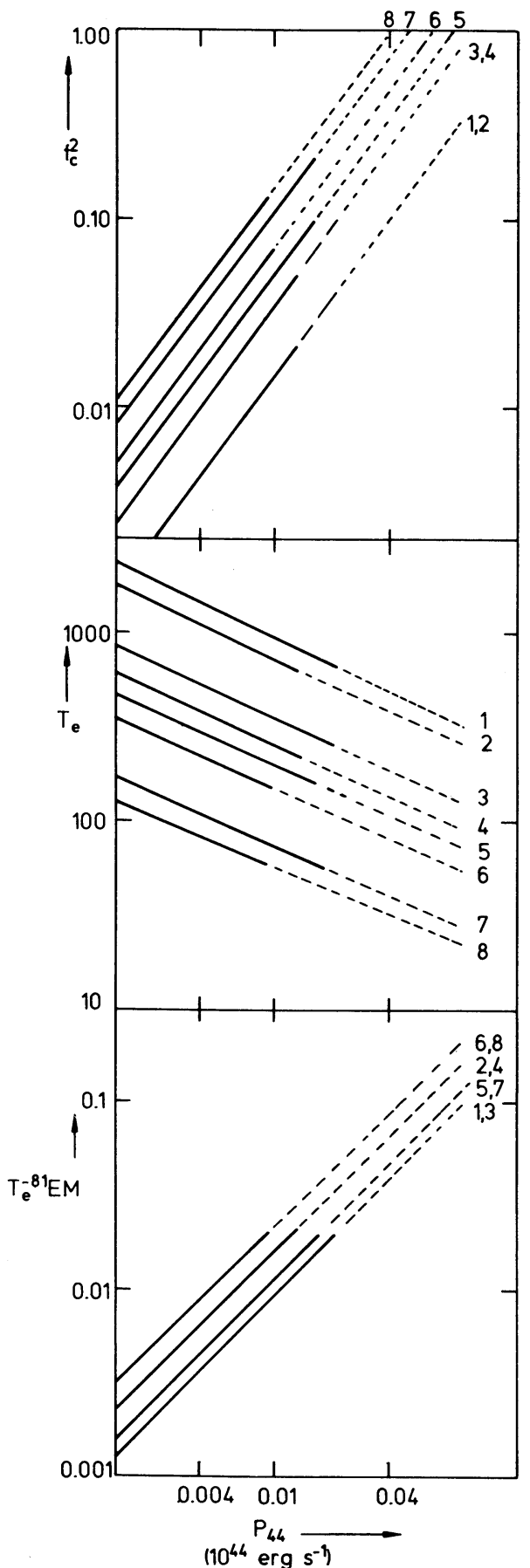

Fig. 6.-Electron temperatures $T_{e}$, clump surface filling factors $f_{c}^{2}$, and constraining parameter $T_{e}^{-0.81} \mathrm{EM}$ as a function of ionizing power available $\boldsymbol{P}_{\mathbf{4 4}}$ for a variety of input assumptions in the clumpy model ( $(\mathrm{III} e$ ). Range of parameter values beyond those admitted by observed limits on $T_{e}^{-0.81} \mathrm{EM}$ is indicated by dashed lines. Cases illustrated are: $1,2: h_{\mathrm{NT}}=3 \mathrm{kpc},\left\langle n_{e}\right\rangle=0.07$; 3, 4: $h_{\mathrm{NT}}=1 \mathrm{kpc},\left\langle n_{e}\right\rangle=0.07 ; 5,6: h_{\mathrm{NT}}=3 \mathrm{kpc},\left\langle n_{e}\right\rangle=0.03 ; 7,8: h_{\mathrm{NT}}=1$ kpc, $\left\langle n_{e}\right\rangle=0.03$; odd numbers assume $a=b=1$; even numbers assume $a=b=0.8$.

With these constraints, we find acceptable solutions only for $P_{44} \leq 0.02$, in rather good agreement with the independent Galactic estimate. If $\left\langle n_{e}\right\rangle \geq 0.07 \mathrm{~cm}^{-3}$, we find $F_{5}^{\prime} / P_{44} \geq 0.4$. This limit is relaxed by assuming lower values for $\left\langle n_{e}\right\rangle$ for example, with $\left\langle n_{e}\right\rangle=0.03 \mathrm{~cm}^{-3}$, we obtain $F_{5}^{\prime} / P_{44} \geq 0.25$.
Clump electron temperatures $T_{e}$, surface filling factors $f_{c}^{2}$, and the parameter $T_{e}^{-0.81}$ EM are graphically shown in Figure 6. From this figure, it is clear that electron temperatures must again be rather low, unless an unrealistically small amount of ionizing input power is assumed. In the face-on galaxy, surface filling factors are of order a few percent. These results imply furthermore mean clump electron densities $0.5<n_{e}<2 \mathrm{~cm}^{-3}$ for $\left\langle n_{e}\right\rangle=0.07-0.10 \mathrm{~cm}^{-3}$, and about $0.1-0.3 \mathrm{~cm}^{-3}$ for $\left\langle n_{e}\right\rangle=0.03 \mathrm{~cm}^{-3}$.

Although this analysis yields only approximate values, we conclude that for a clumpy distribution, the nonthermal emitting and thermal absorbing gas in the mean sample galaxy can be described as follows. The volume filling factor $f_{c}^{3}$ is of order 0.001 , the ionizing input power is of order $10^{42} \mathrm{ergs} \mathrm{s}^{-1}$, and the contribution of the gas absorbing at low frequencies to the total thermal emission at $5 \mathrm{GHz}$ is negligible. Mean line-ofsight electron densities should be of order $0.07-0.10 \mathrm{~cm}^{-3}$, so that individual clump densities are of order $1 \mathrm{~cm}^{-3}$. In that case, electron temperatures are at most $1500 \mathrm{~K}$, and more probably $500-1000 \mathrm{~K}$. If actual line-of-sight densities are lower, e.g., $\left\langle n_{e}\right\rangle \approx 0.03 \mathrm{~cm}^{-3}$, both clump electron densities and temperatures will have to be considerably lower, of order $0.2 \mathrm{~cm}^{-3}$ and $200 \mathrm{~K}$, respectively. The total clump mass is a strong function of the value assumed for $P_{44}$. For $P_{44}=0.02$, masses are of order $10^{8} M_{\odot}$, i.e., a few percent of the total $\mathrm{H} \mathrm{I}$ mass. For $P_{44}=0.01$, the amounts are a factor of 10 less. Here we note again that the above is a simplified picture, because we have assumed identical clumps. In fact, the observed inclination dependence of the ratio $\left(S_{\text {obs }} / S_{\text {extr }}\right.$ corr (Fig. 2$)$ suggests that indeed clumps of lower density with larger volume filling factors become increasingly important with increasing galaxy tilt.

Thus, the model of a clumpy interstellar medium in which nonthermal and thermal plasmas are spatially related yields results consistent with observational limits. Most importantly, for any combination of model parameters, the absorbing thermal material has to be of very low electron temperature.

\section{DISCUSSION}

The question of whether a similar ionized component exists in the Galaxy is of great interest. As we shall see, there is at present no compelling evidence indicating that this is so. However, the Sun's location in a hot and tenuous "Local Bubble" (see Cox and Reynolds 1987) and in the Galactic plane, which is dominated by higher-density, higher temperature ionized regions, complicates matters considerably, so that a final answer cannot yet be given.

First, we consider direct evidence for ionized gas with low electron temperatures. Shaver et al. (1983) studied electron temperatures and metal abundances of fully ionized "classical" $\mathrm{H}$ II regions. They found a radial temperature gradient across the Galactic disk $\left(T_{e}=4000-6000 \mathrm{~K}\right.$ at radii $R_{G}=4-6 \mathrm{kpc}$ ); they ascribe both the low electron temperatures in the inner Galaxy and the gradient itself to a metallicity increase toward the center of the Galaxy. Garay and Rodriguez (1983) found that low-density $\left(n_{e}=25 \mathrm{~cm}^{-3}\right) \mathrm{H} \mathrm{II}$ regions have electron temperatures of order $100 \mathrm{~K}$ lower than high-densiy $\left(n_{e}=10^{3} \mathrm{~cm}^{-3}\right) \mathrm{H}$ II regions. They suggest that the difference is caused by increasing collisional de-excitation at higher electron densities. The strong dependence of $T_{e}$ on abundance and density is demonstrated in the galactic disk model calculations by Collin-Souffrin and Joly (1976). A mod- 
erate increase of abundances over solar neighborhood values at densities of order $n_{e}=1 \mathrm{~cm}^{-3}$ would rapidly lead to equilibrium temperatures of order $T=2000 \mathrm{~K}$ or less.

Studies of Galactic radio recombination line studies are also of some relevance. Lockman $(1980,1981)$ reviewed the observations of radio recombination line $(\mathrm{H} 166 \alpha)$ emission from "blank" regions in the Galactic plane (Solar Neighborhood) and concludes that there is "a broadly distributed medium that extends over a few hundred parsecs" and that has $T_{e}<$ $3000 \mathrm{~K}$, a density of order $n_{e}=1 \mathrm{~cm}^{-3}$, and EM $<1400 \mathrm{pc}$ $\mathrm{cm}^{-6}$. He considers electron temperatures below $500 \mathrm{~K}$ to be "firmly excluded" and generally finds no detectable recombination line emission from random areas of the Galactic plane outside the Solar Circle. More recent observations of $\mathrm{H} 272 \alpha$ emission of "blank" regions enabled Anantharamaiah (1985) to obtain estimates of $T_{e}$ and EM of the emitting gas. Although he favors generally higher values for $T_{e}$, four out of the six lower limits derived by him are close to the low values derived by us for the extragalactic sample.

Dulk and Slee (1975) attribute low-frequency turnovers $\left(v_{t}<100 \mathrm{MHz}\right)$ in Galactic SNR radio spectra to free-free absorption in the line of sight. They give a mean value of the quantity $\left(\mathrm{EM} \times T_{e}^{-1.35}\right)=0.013$ for a mean line of sight of $13.8 \mathrm{kpc}$ and conclude that absorption by relatively cool, partially ionized gas seems most compatible with their observations. For $n_{e}=0.1 \mathrm{~cm}^{-3}$, their result implies $T_{e}=1000 \mathrm{~K}$, which is consistent with the result from $\S$ III $e$. However, our edge-on disk should have an optical depth $\tau_{57.5}>2$, whereas the Dulk and Slee (1975) results yield only $\tau_{57.5} \approx 0.6$. The Sgr A result requires $T_{e} \leq 2000 \mathrm{~K}$ and is the only one associated with an appreciable optical depth $\tau_{57.5}=11$. If the Galaxy is comparable to our extragalactic sample, the only way to reconcile our result with that obtained by Dulk and Slee (1975) appears to be the assumption that the plane of the Galaxy is mostly free from $500-1000 \mathrm{~K}$ ionized gas.

Measurements of emission at very low frequencies (1-100 $\mathrm{MHz}$ ) toward the Galactic poles (i.e., sampling the local Galactic disk) show a turnover at about $3 \mathrm{MHz}$ (Novaco and Brown 1978; Cane 1979), indicating a negligibly small optical depth (face-on) at $57.5 \mathrm{MHz}$. This rules out the presence of significant amounts of 500-1000 K ionized gas in the Solar Neighborhood. Around $10 \mathrm{MHz}$, emission from the Galactic center region is less than 2 times the emission from the Galactic poles and the anticenter (in a $35^{\circ}$ beam; Novaco and Brown 1978). This contrast increases rapidly at higher frequencies, and inspection of the central emission in maps up to $408 \mathrm{MHz}$ (see Haslam et al. 1981, and references therein) suggests the presence, on large angular scales, of another spectral turnover around $150 \mathrm{MHz}$. If this is confirmed, the central Galactic radio spectrum would be similar to that of M33 (Israel, Mahoney, and Howarth 1989), and at least qualitatively similar to that of the extragalactic sample which is the subject of this paper. It would indicate that (as in M33) the absorbing gas fills most, but not all, of the nonthermally emitting volume $(a \approx 0.9)$.

As stated before, we conclude that at present there is no compelling evidence that our Galaxy contains significant amounts of relatively cool ionized gas, as seems to be needed to explain our low-frequency measurements of other galaxies. If this gas is present in our Galaxy, it appears to avoid the solar neighborhood and probably also the Galactic plane (thin disk) itself. It should then be located in the thick disk, which contrib- utes about $90 \%$ of the total power at $408 \mathrm{MHz}$ (Beuermann, Kanbach, and Berkhuysen 1985). Note that such a low-density, low-electron temperature ionized gas filling a large fraction of the nonthermally emitting volume should not be confused with the warm ionized medium (WIM), reviewed, e.g., by Kulkarni and Heiles (1987). This is a substantially ionized diffuse medium with $T_{e} \approx 8000 \mathrm{~K}$, extensively observed by, e.g., Reynolds (1985).

In the preceding, we have shown that photoionization of a (clumpy) low-density medium may explain our observations. However, as an alternative, we mention briefly the interesting time-dependent model constructed by Bania and Lyon (1980). They simulated the effects of OB stars on the physical state of the interstellar medium and found the time-averaged temperature of the intercloud gas to be $1500-2200 \mathrm{~K}$. This gas should be highly clumped. Depending on model parameters, the ionization fraction is of order $0.1-0.2$, and $n_{e}$ is about 0.05 $0.1 \mathrm{~cm}^{-3}$. Clearly, models of this type need further investigation in the context of the observations presented in this paper.

\section{CONCLUSION}

In a survey of $57.5 \mathrm{MHz}$ emission from a large sample of galaxies, we have isolated a subsample disk-dominated spiral galaxies. Their observed flux densities are systematically lower than expected from an extrapolation of higher frequency measurements, which we have interpreted as the effect of free-free absorption of nonthermal emission by thermal material in the galaxy disks. Simple models of such disks serve to show that discrete $\mathrm{H}$ II regions cannot be responsible for the implied absorption, mainly because their filling factor is too small. Thermal gas smoothly distributed throughout the sample galaxies can explain the absorption if it fills a relatively large volume fraction and has a very low electron temperature $\left(T_{e} \approx\right.$ $50 \mathrm{~K})$. Clumps of nonthermal plasma, well mixed with thermal absorbing material, explain the observations for electron temperatures $200 \mathrm{~K} \leq T_{e} \leq 1500 \mathrm{~K}$ (probably $T_{e}=500-1000 \mathrm{~K}$ ) and mean line-of-sight electron densities $n_{e}=0.07-0.10 \mathrm{~cm}^{-3}$. In both cases, the mass of the cool ionized gas is at most a few percent of the $\mathrm{H}$ I mass. Galactic observations show that such a gas is unlikely to be present in the solar neighborhood. If present in other parts, it should be located in the "thick disk" component, rather than in the "thin disk" (Galactic plane). Direct evidence for its existence is, however, at present lacking. Leaving open the question of our own Galaxy, the presence of $a$ (clumpy) ionized phase of low electron density and temperature in the interstellar medium does appear to be a general property of spiral galaxies.

The relatively small emerging fraction in edge-on galaxies $\left(35 \%\right.$ if $\left.F_{5}=0.2\right)$ implies that nonthermal halos are not a major constituent of spiral galaxies, especially if "thick disk" emission is common.

It is a pleasure to thank the staff of Clark Lake Radio Observatory for their support of the observations described in this paper, We also thank T. Bania, J. M. Dickey, W. C. Erickson, H. J. Habing, and P. Katgert for useful comments on various drafts of this paper. In particular, thanks are due to an anonymous referee whose comments led to significant improvements. Part of this work was supported by the National Science Foundation under grant AST 82-15463. 


\section{APPENDIX}

In order to interpret the $57.5 \mathrm{MHz}$ observations of disk-dominated spiral galaxies, we consider two simple models. Model 1 assumes the nonthermal emission to originate from a cylinder with thickness $h_{\mathrm{NT}}$ and radius $r_{\mathrm{NT}}=0.5 f D_{25}$, in which $D_{25}$ is the (corrected) optical galaxy diameter down to a surface brightness of 25th magnitude in $B$ given in the Second Reference Catalog (de Vaucouleurs, de Vaucouleurs, and Corwin 1976). The factor $f$ defines the ratio between (effective) radio and optical diameters. Thermal emission and consequently free-free absorption arises in a number of discrete $\mathrm{H}$ il regions, all characterized by the same emission measure EM. Seen face-on, a fraction $a^{\prime 2}$ of the galactic disk is covered by these $\mathrm{H}$ II regions, so that $0<a^{\prime 2}<1$. (If $a^{\prime 2}>1$, model 1 in fact reduces to model 2; see below). These $\mathrm{H}$ II regions are on average located at a depth of $0.5 h_{\mathrm{NT}}$. If each $\mathrm{H}$ il region absorbs nonthermal emission passing through it with optical depth, the ratio between observed emission and absorption-free (extrapolated) emission is

or

$$
S_{\mathrm{obs}} / S_{\mathrm{extr}}=1-0.5 a^{2}\left(1-e^{-\tau}\right)
$$

$$
a^{2}=2\left(1-S_{\text {obs }} / S_{\text {extr }}\right) /\left(1-e^{-\tau}\right) .
$$

Model 2 assumes again the nonthermal emission to arise in cylinder with thickness $h_{\mathrm{NT}}$ and radius $r_{\mathrm{NT}}=0.5 f D_{25}$, while thermal emission and free-free absorption originate in an embedded cylinder with radius $r_{\mathrm{T}}=a r_{\mathrm{NT}}$ and thickness $h_{\mathrm{T}}=b h_{\mathrm{NT}}$. The plasma is distributed homogeneously throughout both cylinders. Part of the nonthermal emission reaches the observer without suffering absorption on the way, part of it travels through the thermal plasma and suffers absorption $e^{-\tau}$, and part originates in the thermal plasma volume itself, and reaches the observer with absorption $\left(1-e^{-\tau}\right) / \tau$. For the face-on case, we find

$$
S_{\text {obs }} / S_{\text {extr }}=1-a^{2}+0.5 a^{2}(1-b)\left(1+e^{-\tau}\right)+a^{2} b\left(1-e^{-\tau}\right) / \tau \text {. }
$$

Special cases are $a=1$ (equal cylinder radius for thermal and nonthermal emission); $b=1$ (equal cylinder thickness for thermal and nonthermal emission); and $a=b$ (scaled cylinders). The edge-on case yields a more complicated expression:

$$
S_{\mathrm{obs}} / S_{\mathrm{extr}}=1-b g(a)+0.5 b\left[g(a)-a^{2}\right]\left(1+e^{-\tau}\right)+a^{2} b\left(1-e^{-\tau}\right) / \tau,
$$

in which $g(a)=1-\{2 \arccos (a)-\sin [2 \arccos (a)]\} / \pi$. If $S_{\mathrm{obs}} / S_{\text {extr }}$ are known for both cases, cylinder thickness to diameter ratios $h_{T} / 2 r_{T}=0.64 \tau_{\text {face-on }} / \tau_{\text {edge-on }}$ and $h_{\mathrm{NT}} / 2 r_{\mathrm{NT}}=0.64 a / b \tau_{\text {face-on }} / \tau_{\text {edge-on }}$ follow.

As discussed in the text ( $\left(\right.$ III $a$ ), $S_{\text {obs }} / S_{\text {extr }}$ must be corrected for the influence of thermal emission on the spectral index $\alpha$ in the $1-5$ $\mathrm{GHz}$ range. For the mean high-frequency spectral index $\alpha=-0.75$, and a $5 \mathrm{GHz}$ thermal fraction $F_{5}<0.5$ (see Paper I), the correction factor to $S_{\mathrm{obs}} / S_{\text {extr }}$ is almost linearly related to this thermal fraction. Hence, $\tau_{\mathrm{obs}}$ is determined with only $a, b$, and $F_{5}$ as free parameters. Limits to $a$ and $b$ can be obtained from the limiting case $\tau_{\text {obs }}=\infty$.

Allen, R. J., Baldwin, J. E., and Sancisi, R. 1978, Astr. Ap., 62, 397.

Anantharamaiah, K. R. 1985, J. Ap. Astr., 6, 203.

Baars, J. W. M., Genzel, R., Pauliny-Toth, I. I. K., and Witzel, A. 1977, Astr. Ap., 61, 99.

Bania, T., and Lyon, J. G. 1980, Ap. J., 239, 173.

Beuermann, K., Kanbach, G., and Berkhuysen, E. M. 1985, Astr. Ap., 153, 17.

Bregman, J. N., and Harrington, P. J. 1986, Ap. J., 309, 833.

Cane, H. V. 1979, M.N.R.A.S., 189, 465.

Collin-Souffrin, S., and Joly, M. 1976, Astr. Ap., 53, 213.

Cox, D. P., and Reynolds, R. J. 1987, Ann. Rev. Astr. Ap., 25, 303.

Cruz-Gonzalez, C., Recillas-Cruz, E., Costero, R., Peimbert, M., and TorresPeimbert, S. 1974, Rev. Mexicana Astr. Af., 1, 211.

de Bruyn, A. G., Crane, P. C., Price, R. M., and Carlson, J. B. 1976, Astr. Ap., 46, 243.

Deharveng, J. M., and Pellet, A. 1970, Astr. Ap., 9, 181.

de Vaucouleurs, G., De Vaucouleurs, A., and Corwin, H. G. 1976, Second Reference Catalog of Bright Galaxies (Austin: University of Texas Press).

Dulk, G. A., and Slee, O. B. 1975, Ap. J., 199, 61.

Emerson, D. T., Kronberg, P. P., and Wielebinski, R. 1978, Astr. Ap., 63, 49.

Erickson, W. C., and Fisher, J. R. 1974, Radio Sci., 9, 387.

Erickson, W. C., Mahoney, M. J., and Erb, K. 1982, Ap. J. Suppl., 50, 403.

Falgarone, E., and Perault, M. 1988, Astr. Ap., 205, L1

Garay, G., and Rodriguez, L. F. 1983, Ap. J., 266, 263.

Gayet, R. 1970, Astr. Ap., 9, 312

Gioia, I. M., Gregorini, L., and Klein, U. 1982, Astr. Ap., 116, 164.

Hanbury Brown, R., and Hazard, C. 1951, M.N.R.A.S., 111, 357

Harnett, J. I., and Reynolds, J. E. 1985, M.N.R.A.S., 215, 247.

Haslam, C. G. T., Salter, C. J., Stoffel, H., and Wilson, W. E. 1981, Astr. Ap. Suppl., 47, 1 .

Hawley, S. A. 1978, Ap. J., 224, 417

Haynes, R. F., Huchtmeier, W. K. H., Siegman, B. C., and Wright, A. E. 1975, A Compendium of Radio Measurements of Bright Galaxies (Sydney: C.S.I.R.O. Div. Radiophys.).

Hodge, P. W., and Kennicutt, R. C. 1983, A.J., 88, 296

Hummel, E. 1980, Astr. Ap. Suppl., 41, 151 .

Israel, F. P. 1980, Astr. Ap., 90, 246.

\section{REFERENCES}

Israel, F. P., Goss, W. M., and Allen, R. J. 1975, Astr. Ap., 40, 421

Israel, F. P., Mahoney, M. J., and Howarth, N. 1989, Astr. Ap., submitted.

Israel, F. P., and van der Hulst, J. M. 1983, A.J., 88, 1736 (Paper I).

Israel, F. P., and van der Kruit, P. C. 1973, Astr. Ap., 32, 363.

Kellermann, K. I., and Pauliny-Toth, I. I. K. 1969, Ap. J. (Letters), 155, L71.

Kuhr, H. 1980, Ph.D. thesis, University of Bonn.

Kulkarni, S. R., and Heiles, C. 1987, in Interstellar Processes, ed. H. Thronson and D. Hollenbach (Dordrecht), p. 87.

1988, in Galactic and Extragalactic Astronomy, ed. G. L. Verschuur and K. Kellerman (San Francisco) p. 95.

Kwitter, K. B., and Aller, L. H. 1981, M.N.R.A.S., 195, 939.

Lockman, F. J. 1980, in Radio Recombination Lines, ed. P. A. Shaver (Dordrecht: Reidel), p. 185.

. 1981, in The Phases of the Interstellar Medium, ed. J. M. Dickey (Green Bank: NRAO), p. 127.

Martin, P. G. 1988, Ap. J. Suppl., 66, 125.

Mezger, P. G., and Henderson, A. P. 1967, Ap. J., 147, 471.

Monnet, G. 1974, in Galactic Radio Astronomy, ed. F. J. Kerr and S. C. Simonson (Dordrecht: Reidel), p. 249.

Novaco, J. C., and Brown, L. W. 1978, Ap. J., 221, 114.

Oster, L. 1961, Rev. Mod. Phys., 33, 525.

Panagia, N., and Terzian, Y. 1984, Ap. J., 287, 315.

Reynolds, R. J. 1985, Ap. J., 294, 256.

Shaver, P. A., McGee, R. X., Newton, L. M., Danks, A. C., and Pottasch, S. R. 1983, M.N.R.A.S., 204, 53 .

Shields, G. A., and Searle, L. 1978, Ap. J., 222, 821.

Sivan, J. P. 1974, Astr. Ap. Suppl., 16, 163.

Slee, O. B. $1972 a$, Ap. Letters, $12,75$.

1972b, Proc. Astr. Soc. Australia, 2, 159

1977, Australian J. Phys., Ap. Suppl., 43, 1.

Smith, H. E. 1975, Ap. J., 199, 591.

Torres-Peimbert, S., Lazcano-Araujo, A., and Peimbert, M. 1974, Ap. J., 191,

van der Kruit, P. C. and Allen, R. J. 1976, Ann. Rev. Astr. Ap., 14, 417

Weisberg, J. M., Boriakoff, V., and Rankin, J. 1979, Astr. Ap., 77, 204

F. P. IsRAEL: Sterrewacht, Huygens Laboratory, Postbus 9513, 2300 RA Leiden, The Netherlands

M. J. Mahoney: Jet Propulsion Laboratory, Mail Stop 168-327, 4800 Oak Grove Drive, Pasadena, CA 91109 